\title{
Quality and denoising in real-time fMRI neurofeedback: a methods review
}

\author{
Stephan Heunis ${ }^{\mathrm{a}, \mathrm{b}, *}$, Rolf Lamerichs ${ }^{\mathrm{a}, \mathrm{b}, \mathrm{c}}$, Svitlana Zinger ${ }^{\mathrm{a}, \mathrm{b}}$, Cesar Caballero-Gaudes ${ }^{\mathrm{d}}$, \\ Jacobus FA Jansen ${ }^{\mathrm{a}, \mathrm{e}, \mathrm{f}}$, Bert Aldenkamp ${ }^{\mathrm{a}, \mathrm{b}, \mathrm{f}, \mathrm{g,h}}$ and Marcel Breeuwer ${ }^{\mathrm{i}, \mathrm{j}}$ \\ ${ }^{a}$ Department of Electrical Engineering, Eindhoven University of Technology, Eindhoven, The Netherlands \\ ${ }^{\mathbf{b}}$ Department of Research and Development, Epilepsy Centre Kempenhaeghe, Heeze, The Netherlands \\ c Philips Research, Eindhoven, The Netherlands \\ d Basque Center on Cognition, Brain and Language, San Sebastian, Spain \\ e Department of Radiology, Maastricht University Medical Centre, Maastricht, The Netherlands \\ ${ }^{f}$ School for Mental Health and Neuroscience, Maastricht, The Netherlands \\ ${ }^{g}$ Laboratory for Clinical and Experimental Neurophysiology, Neurobiology and Neuropsychology, Ghent \\ University Hospital, Ghent, Belgium \\ ${ }^{\mathbf{h}}$ Department of Neurology, Maastricht University Medical Center, Maastricht, The Netherlands \\ i Department of Biomedical Engineering, Eindhoven University of Technology, Eindhoven, The Netherlands \\ j Philips Healthcare, Best, The Netherlands \\ * Corresponding author \\ Email: j.s.heunis@tue.nl \\ Address: PO Box 513; Flux building, room 5.084; Eindhoven; Noord-Brabant 5600MB; NL
}

\section{$1 \quad$ Abstract}

2 Neurofeedback training using real-time functional magnetic resonance imaging (rtfMRI-NF) allows subjects voluntary control of localized and distributed brain activity. It has sparked increased interest as a promising non-invasive treatment option in neuropsychiatric and neurocognitive disorders, although its efficacy and clinical significance are yet to be determined. Maximization of neurofeedback learning effects in accordance with operant conditioning requires the feedback signal to be closely contingent on real brain activity, necessitating the use of effective real-time denoising methods to prevent sham feedback. In this work, we present the first extensive review of acquisition, processing and quality control methods available to improve the quality of the neurofeedback signal. Furthermore, we investigate the state of denoising and quality control practices in 128 recently published rtfMRI-NF studies. We found: (i) that less than a third of the studies reported implementing standard real-time fMRI denoising steps; (ii) significant room for improvement with regards to methods reporting; and (iii) the need for methodological studies quantifying and comparing the contribution of denoising steps to the neurofeedback signal quality. Advances in rtfMRI-NF research depend on reproducibility of methods and results. To this end, we recommend that future rtfMRI-NF studies: (i) report implementation of a set of standard realtime fMRI denoising steps according to a proposed COBIDAS-style checklist (https://osf.io/kjwhf/); (ii) ensure the quality of the neurofeedback signal by calculating and reporting community-informed quality metrics and applying offline control checks; and (iii) strive to adopt transparent principles in the form of methods and data sharing and support of open-source rtfMRI-NF software.

Keywords: Real-time, fMRI, neurofeedback, denoising, quality, reproducibility 


\section{Introduction}

Real-time functional magnetic resonance imaging (rtfMRI) involves the dynamic processing, analysis and visualization of a subject's changing blood oxygen level-dependent (BOLD) signal and related information while the subject is inside the MRI scanner. It was initially proposed and developed by Cox et al. (1995) as a tool for real-time data quality monitoring, functional activation mapping and interactive experimental design. Since its inception the technology has expanded to include a variety of software tools that allow pre-experimental and pre-surgical functional localization (Hirsch et al., 2000; Binder, 2011), real-time functional activity mapping (as is available in the software accompanying MRI systems from all major vendors), brain computer interfacing (e.g. Sorger et al., 2012), brain state decoding (LaConte, 2011), real-time neurofeedback (Sitaram et al., 2017), and teaching (Weiskopf et al., 2007b).

Neurofeedback training as an application of real-time fMRI (rtfMRI-NF) has gained much interest in the past decade due to its ability to help subjects achieve learned regulation of regional brain activation, as was initially demonstrated by Yoo and Jolesz (2002) in a motor task experimen. Interested readers are referred to Sitaram et al. (2017) for a recent review of rtfMRI-NF functionality, technology and applications. Shortly, by feeding a representation of quantified brain activity back to the subject in the scanner in near-real-time, and asking subjects to increase or decrease the presented metric by adopting one of several possible training strategies (or none at all), subjects have been able to regulate their own BOLD signal. This is evidenced by increased activation levels and cluster sizes in the areas of interest measured over multiple training sessions (see for example DeCharms, 2007).

In further steps, learned brain activity regulation through neurofeedback training has been used in neuropsychological and psychiatric disorders to test for behavioural correlates, aiming to investigate non-invasive rtfMRI-NF as an alternative to more invasive treatment modalities like pharmacological interventions, surgery or deep brain stimulation. Several studies have reported significantly beneficial behavioural, symptomatic or experiential changes after rtfMRI-NF training in a variety of clinical or other populations, including major depressive disorder (Linden et al., 2012), tinnitus (Emmert et al., 2017), attention deficit and hyperactivity disorder (Alegria et al., 2017), obesity (Spetter et al., 2017), and nicotine cravings (Canterberry et al., 2013).

Evidence for widespread and clinically significant effects of rtfMRI-NF training has however been called into question by recognizing a lack of replication studies (Sulzer et al., 2013a), of blinded placebo-controlled study designs (Thibault et al., 2018) and of reproducible methods (Stoeckel et al., 2014). In order for rtfMRI-NF to advance as a research and possibly clinical tool, reproducibility of methods, of results and of inferences are imperative ${ }^{1}$ (Goodman et al., 2016; Munafo et al., 2017). Central to several aspects influencing the reproducibility of both results and methods in rtfMRI-NF is the concept of quality, pertaining to real-time fMRI data, to the neurofeedback signal and to methods reporting.

[1] To prevent ambiguous interpretations of the more general terms "reproducibility", "replicability" and "repeatability", in this work we adhere to the definitions for methods-, results- and inferential reproducibility in the biomedical sciences proposed by Goodman et al. (2016). 
Operant conditioning (Skinner, 1945) has been postulated to be a learning model underlying neurofeedback training (Weiskopf et al., 2004b; Sitaram et al., 2017). To maximize learning effects in this model, the reward or stimulus (i.e. reinforcement) presented to the subject needs to be contingent on the subject's behaviour. In rtfMRI-NF the implication is that the neurofeedback signal calculated from the fMRI data acquired in real-time should aim to represent true brain activity relating to the subject's ongoing cognitive processes (Koush et al., 2012). It is well-known that the resting state or task-induced BOLD signal is contaminated by several scanner-, sequence-, subject- or experiment-related nuisance signals and artefacts (Murphy et al., 2014; Power et al., 2014; Caballero-Gaudes and Reynolds, 2016; Liu, 2016). An important concern in rtfMRI-NF is thus that, if these contaminants are not sufficiently accounted for during acquisition or removed through real-time processing, the feedback signal will remain contaminated and will thus not sufficiently reflect brain activity of interest. This may lead to sham learning due to the absence of contingency, or to a nuisance signal being trained during rtfMRI-NF instead of the subject's BOLD response (LaConte, 2011; Koush et al., 2012), which confounds the experiment and produces uninterpretable results.

A potential further concern is that, with the recent fast expansion of this field of research, attention to reproducible methods might be lagging behind the publishing of novel study results. Few studies in this field have meticulously investigated such detail as preprocessing steps, quality control checks, or best practices for methods reporting. As such, adherence to best practices in rtfMRI-NF is unclear, which might lead to incorrect interpretations of the data were meta-analyses to be conducted.

In an effort to move towards improved reproducibility in the field of rtfMRI-NF, this work reviews the methods currently available to the researcher to improve the data quality and signal-to-noise ratio (SNR) of the rtfMRI-NF signal and of real-time fMRI data and studies in general. Specifically, we investigate three main research questions:

1. What are challenges to effective denoising and improving quality in rtfMRI-NF?

2. Which steps have recent rtfMRI-NF studies taken to improve data quality and SNR?

3. Which methods for denoising data and improving data quality and SNR are available to the researcher studying rtfMRI-NF?

To preface addressing these questions, a background on the BOLD signal and its contaminants and on the details of the calculated neurofeedback signal is provided. Although both acquisition and processing methods are covered in this work, focus is given to the latter. We conclude with a general discussion and future recommendations based on the reviewed literature.

\section{Background}

\subsection{The BOLD signal, noise, artefacts and correction methods}

The $\mathrm{T}_{2} *$-weighted BOLD signal typically acquired using standard gradient-echo echo-planar imaging (EPI) in fMRI represents hemodynamic and metabolic responses, through a neurovascular coupling, to alterations in neuronal activity (Ogawa et al., 1998). It results from a complex interaction between neural metabolism, blood oxygen concentration 
121 (specifically the local concentration of paramagnetic deoxyhemoglobin), cerebral blood flow

Given its dependence on neuronal metabolism, cerebral blood flow/volume and the inherent properties of MRI (and the EPI sequence in particular), it should be no surprise that the BOLD signal has several contaminants and remains difficult to isolate as a proxy for true neuronal activity (Diedrichson and Shadmehr, 2005). fMRI is typically plagued by a variety of noise fluctuations and artefacts originating either from the subject, from the experimental conditions, from the inherent properties of the acquisition sequence, or from the scanner and its (interfering) environment.

Much research effort has been given to ridding fMRI of noise. These efforts can be divided into two main categories: acquisition and data processing. Acquisition methods typically entail pulse sequence alterations or MRI parameter choices that improve the BOLD sensitivity, increase SNR, or preempt and minimize the effects of artefacts that may occur during scanning. Data processing methods to remove noise have been widely reported and typically take the form of model-based or model-free methods. Examples of model-based denoising or artefact removal steps in fMRI preprocessing pipelines include: slice-time correction, 3D volume realignment, frequency band filtering, spatial smoothing, distortion correction, outlier removal/scrubbing (Siegel et al., 2014), regression of movement parameter residuals (Friston et al., 1996), global signal regression (Power et al., 2017), and physiological noise regression (Glover et al., 2000; Birn et al., 2006). Model-free methods mainly include the identification and removal of artefacts through the use of spatial independent component analysis (ICA; Perlbarg et al., 2007; Griffanti et al., 2014). For a thorough understanding of fMRI noise and denoising methods, readers are referred to indepth reviews by Murphy et al. (2014), Power et al. (2014), Caballero-Gaudes and Reynolds (2016), Liu (2016), Kundu et al. (2017) and Power et al. (2018).

Studies have investigated the implications of not correcting sufficiently for (or ignoring) fMRI noise, confounds and artefacts. Head motion, for example, has been shown to result in false activity patterns when coupled to the timing of the task paradigm (Hajnal et al., 1994), to cause simultaneous decreases in long-distance correlations and increases in short-distance correlations within functional connectivity networks (Power et al., 2012), and to cause problems in interpretations of functional connectivity measures across groups (Van Dijk et al., 2011). The hemodynamic response function (HRF) is known to vary spatially across the brain, as well as between subjects and between studies (Huettel and McCarthy, 2000, Handwerker et al., 2012), but the time-to-peak in standard task-fMRI experiments is typically assumed to be $\sim 4-6 \mathrm{~s}$ brain-wide. Gitelman et al. (2003) investigated this assumption and showed the importance of deconvolution prior to modeling psychophysiologic interactions when considering functional/effective connectivity measures across the brain. HRF variability was further explored in a recent study by Rangaprakash et al. (2018) which found that, if not accounted for, it could lead to identification of false functional connectivity measures. Noise sources resulting in global signal fluctuations (e.g. respiratory cycles) can also lead to incorrect attribution of signal to brain activity if regional BOLD fluctuations are considered in isolation (Noll and Schneider, 1994), i.e. without regard to possibly confounding global signal correlations.

Noise sources remain problematic whether fMRI data are considered in real-time or offline. It is therefore important when considering real-time fMRI to address these known noise 
fluctuations and artefacts so as to increase the BOLD SNR, and to consider the implications of not correcting for these nuisances.

\subsection{Real-time fMRI}

173

\section{0}

The vast majority of real-time fMRI implementations use single echo echo-planar imaging (EPI) as the preferred acquisition method, likely due to its prevalence in conventional functional imaging. Acquired slices are reconstructed on the MRI scanner hardware, and upon completion each functional image volume is typically shared on a local network from where it is accessible by the real-time fMRI application software. Fig. 1 illustrates a standard real-time fMRI setup, including components of a neurofeedback application.

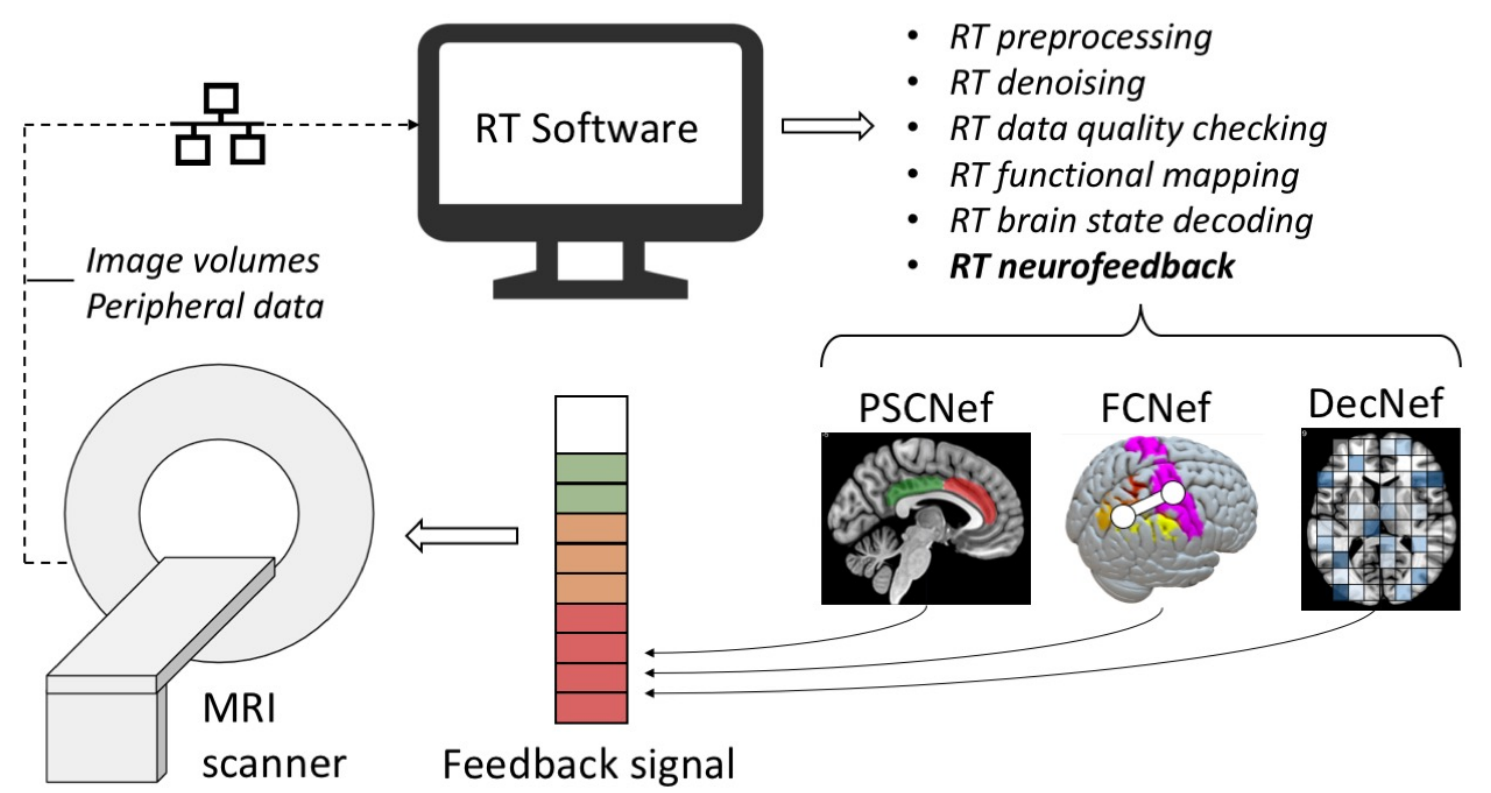

Fig. 1. A typical real-time fMRI technical setup, showing detailed components of a neurofeedback application $(\mathrm{RT}=$ realtime; PSCNef = percentage signal change neurofeedback; FCNef = functional connectivity neurofeedback; DecNef = decoded neurofeedback).

The 'real-time' time frame is loosely defined to be the interval between two successive functional image scans, i.e. the repetition time (TR), indicating that the concept 'real-time' varies according to the application. Ideally, all required processing steps applied to each functional image should be completed sufficiently prior to or by the time the next image in the session is acquired, thus allowing the presentation of up-to-date image information to the researcher and/or subject. Given the nature of the HRF, real-time fMRI not only includes a delay of one TR typically used for data processing (reported to be $\sim 1-3 \mathrm{~s}$ in standard rtfMRI$\mathrm{NF}$ applications), but also a substantial delay due to the indirect measurement of neuronal activity ( 4-6s). As such, typical implementations of real-time fMRI often only allow a representation of brain activity about $5 \mathrm{~s}$ or more after such changes occurred on a neuronal level, leading to the term 'near-real-time'. This definition is distinct from the same term used by some studies to refer to a real-time fMRI processing stream that delivers brain activity and other information within minutes after completing the functional scan session (e.g. Voyvodic, 1999). Note that neurofeedback presentation does not have to be synchronised with image acquisition and can be updated continuously or intermittently depending on the fMRI acquisition rate, software implementation and experimental design. 
The data processing steps necessary to derive a near-real-time representation of brain activity vary according to the application and implemented toolset, but typically follow the course of conventional task-based or resting state fMRI analysis, where data are first preprocessed to remove artefacts or noise fluctuations and then analysed with model-based or model-free statistical methods to extract information of interest. Real-time fMRI neurofeedback preprocessing typically consists of 3D volume realignment, spatial smoothing, linear or polynomial trend removal and temporal filtering, while few applications report the use of slice-timing correction, physiological noise correction methods or real-time distortion correction. These reported preprocessing steps are delineated further in Section 4.

Univariate statistical analysis methods implemented in real-time include recursive correlation between voxel time-series and a reference vector (Cox et al., 1995), t-tests (Voyvodic, 1999), multiple linear regression (Smyser et al., 2001), and general linear model (GLM; Bagarinao et al., 2003). Multivariate methods applied to real-time fMRI are less common, with the realtime implementation of a support vector machine classifier (SVM; Laconte et al., 2007) being the first example, and sparse logistic regression (Shibata et al., 2011) and sparse multinomial or linear regression (Shibata et al., 2016) being used for recent real-time pattern decoding.

To decrease the required per-volume processing time, algorithms generally make use of sliding window (Gembris et al., 2000) or incremental approaches (Bagarinao et al., 2003) when analysing time-series data (see Fig. 2). While time-windowed algorithms allow more sensitivity to temporal brain activity fluctuations by only analysing a recent subset of the acquired data, they are characterised by a decrease in statistical power (Weiskopf et al., 2007b), the converse being the case for incremental or cumulative algorithms that analyse all acquired data. A distinction is made here between incremental methods that use the data in each new iteration to update a growing statistical model so as to avoid recomputation (e.g. the incremental GLM developed by Bagarinao et al., 2003, that incrementally estimates and updates the coefficients of a GLM), and cumulative methods that repeat the operation during each iteration on all data acquired up to that iteration.

In general, real-time fMRI pre-processing and statistical analysis pipelines are simplified versions of their standard offline counterparts because priority is given to fast algorithms (those that converge in as few as possible iterations) and to the inclusion of the minimum sufficient steps to achieve an acceptable level of data quality, so as to decrease per-volume processing time. This tradeoff between maintaining a high level of method accuracy and minimizing the required per-volume processing time has initially been a large constraint to expanding the complexity of real-time fMRI processing steps, but has become increasingly easier to manage with advances in modern computing technology and algorithm development. The use of parallel computing using clusters (e.g. Bagarinao et al., 2003), multiple processing cores (e.g. Koush et al., 2017), and parallel cloud computing (Wang et al., 2016; Cohen et al., 2017), as well as the use of graphical processing units (GPUs; Eklund et al., 2011; Scheinost et al., 2013; Misaki et al., 2015), allow substantial decreases in required per-volume processing times and could accordingly afford real-time fMRI tools a comparative level of complexity and accuracy as that of their offline counterparts. New research avenues become possible like whole-brain real-time fMRI (Misaki et al., 2015), full correlation matrix analysis (Wang et al., 2016), and complex processing for more effective noise removal (Misaki et al., 2015). With such computing power advancements, research outputs become more dependent on how the researcher selects MRI sequence parameters and signal processing steps, and less so on per-volume time restrictions. This shift enables increases in real-time BOLD quality. 


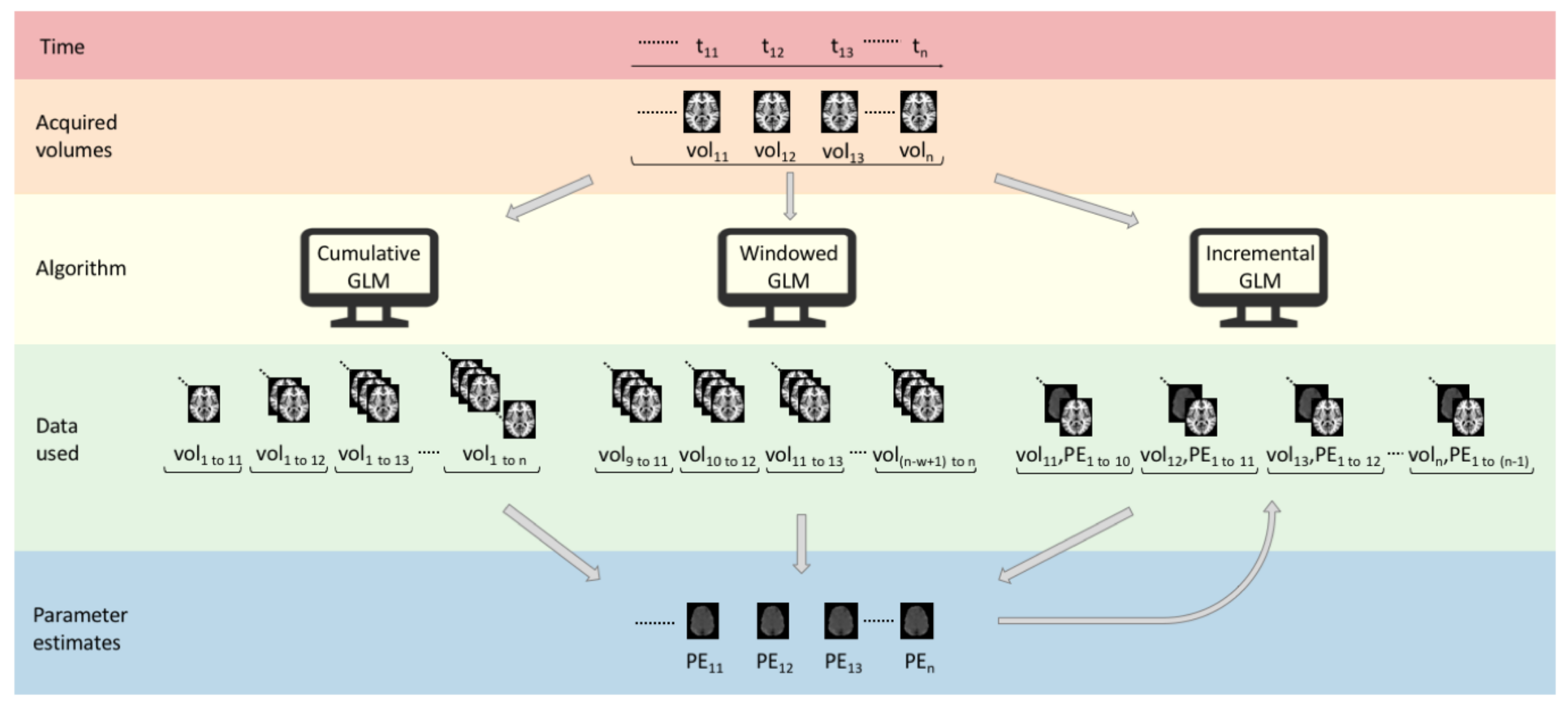

Fig. 2: A representation of the three most commonly used real-time general linear model (GLM) algorithms, indicating the differences in how data available for each iteration are incorporated into the algorithms. A cumulative GLM (cGLM) uses all available data at each iteration to calculate the parameter estimates per iteration. A windowed GLM (wGLM) uses a window size of the most recent $w$ ( $=3$ in this example) volumes to calculate parameter estimates for a specific iteration. An incremental GLM (iGLM) incorporates volume data for each new iteration into an existing state 


\subsection{Real-time fMRI Neurofeedback}

The rtfMRI-NF signal presented to the subject varies per study, but has been based on measures derived through three main computing methods: (1) BOLD activity percentage signal change typically in a single or differential region of interest (PSCNef), (2) functional connectivity between BOLD activity in multiple ROIs (FCNef), and (3) multivariate (or multivoxel) pattern analysis (MVPA), typically within a single ROI (DecNef).

The majority of volunteer and patient rtfMRI-NF studies have used a single or multiple ROI approach to calculate the feedback signal, specifically using the percentage signal change (PSCNef) of the spatially averaged signal obtained from all voxels within the defined ROI(s), as illustrated in Fig. 3. Various regions of interest have been selected for different reasons, with the insula, amygdala, and the cingulate, auditory, visual and motor cortices often forming the basis for neurofeedback (Thibault et al., 2018). Regions of interest are most often acquired using a subject-based functional localizer run before neurofeedback commences (Weiskopf et al., 2007b), although template based or anatomical ROIs have also been used. Several important factors need to be accounted for when using single ROIs as the feedback target. This includes increased signal dropout resulting from EPI imaging of lower or midbrain regions (e.g. the limbic system or medial temporal region) due to increased magnetic susceptibility gradients near air/tissue borders, leading to lower BOLD SNR.

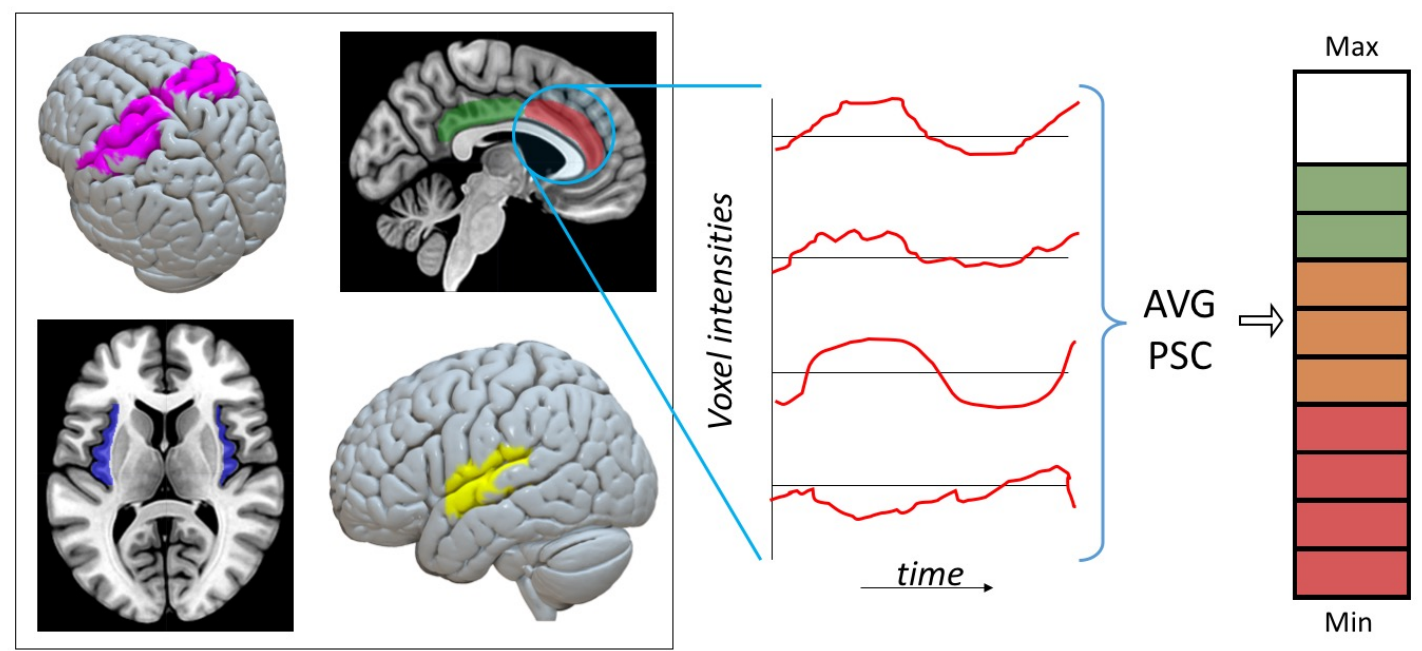

Fig. 3. A linear neurofeedback signal (right) calculated as the average percentage signal change within the anterior cingulate cortex. Examples of other regions of interest are also displayed (left).

Functional connectivity neurofeedback (FCNef, Watanabe et al., 2017) was introduced to target applicable brain networks and their correlation rather than isolated activity in specific ROIs (Ruiz et al., 2013), and it has shown promise as an alternative to PSCNef. The principle is explained in Fig. 4, where the average signal from different ROIs (in this case the motor and parietal cortices) are correlated across a moving time window to calculate the feedback signal. Various connectivity measures can be used as a basis for the neurofeedback signal, including Pearson's Correlation (Zilverstrand et al., 2014) and Dynamic Causal Modeling (Koush et al., 2013). When using FCNef, care has to be taken to prevent global signal fluctuations from biasing the calculated connectivity measure (and thus the feedback signal), based on concerns raised by Power et al. (2012) and Van Dijk et al. (2011) that were highlighted earlier. 


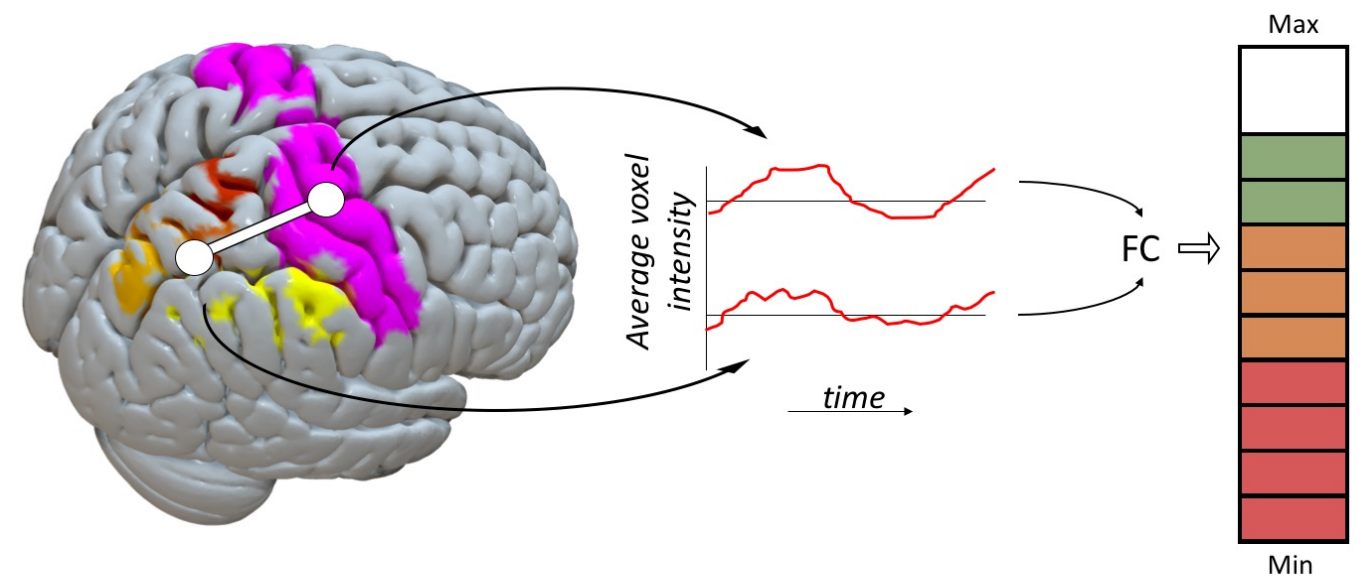

Fig. 4. A linear neurofeedback signal (right) calculated as the functional connectivity (e.g. windowed Pearson's correlation) level between the motor and parietal cortex ROIs.

Real-time fMRI multivoxel pattern analysis (also known as brain state decoding, decoded neurofeedback or DecNef, Watanabe et al., 2017) applies multivariate techniques to fMRI data, first by constructing a decoder using pre-neurofeedback session data with known taskmodulation or states, which is then used in real-time to decode each acquired volume for similarity to the target brain state pattern (see Fig. 5). Support vector machine (SVM) algorithms for real-time classification have been incorporated into several rtfMRI-NF toolboxes (AFNI - LaConte et al., 2007; Turbo-BrainVoyager - Sorger et al., 2010; FRIEND - Basilio et al., 2015). In addition, sparse logistic, sparse multinomial and sparse linear regression algorithms have been often used as decoders, depending on both the software implementation and the nature of the required neurofeedback signal (e.g. binary or linear). For further detail, LaConte (2011) and Watanabe et al. (2017) provide reviews of methodology and studies, respectively, using real-time fMRI DecNef.
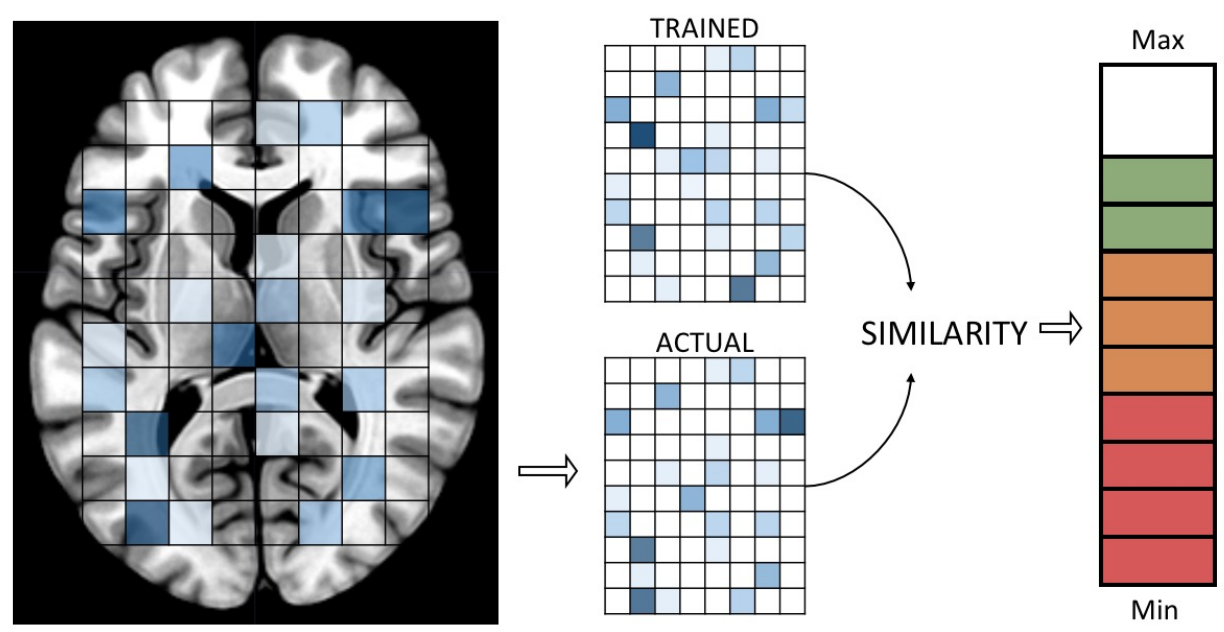

Fig. 5. A linear neurofeedback signal (right) decoded as a representation of the similarity between voxel intensities in the trained pattern (resembling some known brain state) and the real-time brain voxel pattern.

For more examples of studies using the methods above, readers are referred to Watanabe et al. (2017) and Thibault et al. (2018). Watanabe et al. (2017) explored advances in FCNef and DecNef based real-time fMRI, providing a list of 9 studies using these methods, explaining concepts and listing new challenges and possible solutions in the realm of FCNef and DecNef methods. Thibault et al. (2018) conducted a critical systematic review of 99 rtfMRI-NF 
studies (mostly PSCNef) to evaluate the effectiveness of reported experimental protocols to train subjects to self-regulate their BOLD signal.

Apart from the feedback type and target region, several aspects of rtfMRI-NF can influence the ability of subjects to learn self-regulation of the neurofeedback signal (Kadosh and Staunton, 2018). Experiments need to take account of the advantages or disadvantages of, amongst others, the regularity of neurofeedback presentation (continuous vs intermittent), external rewards for learning outcomes, simultaneous visual display of task and feedback information, instructions given to subjects on learning strategy, variability in individual learning strategy of subjects, real-time data quality measures, and the use of control groups and blinding in order to reach the full potential of a rigorously designed and reproducible rtfMRI-NF experiment.

\section{Quality in real-time fMRI neurofeedback}

Quality is an umbrella term that is applicable to real-time fMRI data, to the neurofeedback signal and to the methods reporting process. Generally, fMRI data quality is a measure of how well the acquired BOLD data reflects the signal of interest, i.e. neural activity, and it is influenced by variability in multiple factors including the subject, the experimental design and acquisition (spatial resolution, image contrast, field strength, etc). If fMRI data quality is high, the implication is that signals that are not of interest (i.e. noise) are either absent from or not biasing our interpretation of the processed data, and there is lower possibility of making false inferences. This improves results and inferential reproducibility, and thus scientific progress. As an extension of fMRI data, high quality of the real-time fMRI neurofeedback signal implies that a signal closely reflective of brain activity (and not of noise or artefacts) is fed back to the subject in real-time. Quality in methods reporting implies that a published study contains enough information about the applicable experimental-, acquisition- and data processing steps that would allow different researchers to reproduce the methods. Here, high quality has a direct and beneficial influence on methods reproducibility.

When aiming to improve quality in real-time fMRI neurofeedback it is therefore advised to (1) separate the effects of noise (measurement-, system-, or subject-based) from true BOLD fluctuations, to (2) quantify and report the quality of real-time fMRI data and the calculated neurofeedback signal, and to (3) accurately and sufficiently report the use of applicable realtime fMRI denoising methods.

\subsection{Measuring, comparing and reporting rtfMRI data quality}

Traditionally, apart from expert visual inspection of fMRI datasets to identify low quality volumes / sessions / subjects / sites (as evidenced by visible artefacts in fMRI images like excessive motion, RF interference, or ghosting), the temporal signal-to-noise ratio (tSNR) has been an important quantitative measure of fMRI data quality and the ability of an experiment to find effects of interest (Parrish et al., 2000; Welvaert and Rosseel, 2013; Murphy et al., 2007). tSNR gives an indication of the per-voxel signal fluctuations rated against the background noise fluctuations, with an example equation being:

$t S N R=\frac{\mu}{\sigma}$ 
Here, $\mu$ and $\sigma$ refer to the (per-voxel) mean and standard deviation of the fMRI time series, respectively. A variation of tSNR is the temporal contrast-to-noise ratio (tCNR, Geissler et al., 2007), which investigates the difference between functional contrast conditions (e.g. task activity vs. baseline activity) rather than considering signal fluctuations at all times. As such, tCNR could be defined as (Koush et al., 2012):

$$
\text { tCNR }=\frac{\mu_{\text {contrast }}}{\sigma_{\text {all }}}=\frac{\mu_{\text {condition }}-\mu_{\text {baseline }}}{\sqrt{\sigma^{2}}}=\left(\frac{\Delta S}{\mu} * t S N R\right)
$$

The part of Eq.2 in brackets provides a common definition of CNR (Krüger et al., 2001), where $\Delta S$ is the signal change due to an experimental condition. Eq.2 thus assumes that $\mu_{\text {condition }}=\Delta S$, and that $\mu_{\text {baseline }}=0$.

A simple fMRI quality inspection approach could be to compare the tSNR or tCNR values calculated before and after denoising to see if the change brings about a data quality increase. It should be noted that, depending on how noise and signal sources are defined spatially and on the type of condition and baseline choices, tSNR and tCNR values could vary and are not automatically standardized. Importantly, there is little consensus on a standard definition of tSNR and tCNR (Welvaert and Rosseel, 2013), which could hinder comparability between different sites and studies. Additionally, a single metric is unlikely to provide a full quantitative view of the quality of a complex signal such as fMRI, and further measures could be insightful.

Historically, AFNI's real-time fMRI module (Cox et al. 1995) supported the ability to display motion parameters to the subject in order the suppress head motion (Yang et al., 2005) and to feed back a display of variability in areas affected by physiological noise (e.g. ventricles) in order to reduce the standard deviation of the fMRI signal (Bodurka et al., 2009). These parameters can inherently also serve as real-time quality indicators.

More recent real-time quality tools include Framewise Integrated Real-time fMRI Monitoring (FIRMM; Dosenbach et al., 2017), which focuses on real-time motion tracking and related quality metrics, and rtQC, a recently presented open-source collaborative framework for quality control methods in real-time (Hellrung et al., 2017, Heunis et al., 2019a). rtQC currently focuses on highlighting quality issues between the offline and real-time variants of fMRI data as well as real-time visualisation of quality control metrics, including a real-time display of a grayplot (a 2D representation of voxel intensity fluctuations over time; Power, 2017).

In further rtfMRI-NF literature, studies employing data quality checks focus on pre- and postreal-time application of quality control processes. Stoeckel et al. (2014) propose the calculation and use of tSNR and the concordance correlation coefficient on pilot data to determine, respectively, whether the rtfMRI-NF signal is detectable and reproducible between runs. They also proposed a list of 7 high-level guidelines to help optimize real-time fMRI neurofeedback for therapeutic discovery and development: (i) the rtfMRI signal is accurate and reliable, (ii) rtfMRI neurofeedback leads to learning, (iii) the training protocol is optimized for rtfMRI-based neurofeedback and learning, (iv) there is an appropriate test of training success, (v) rtfMRI neurofeedback leads to behavioral change, (vi) an appropriate rtfMRI neurofeedback-based clinical trial design is in place, and (vii) sharing resources and using common standards. Sorger et al. (2018) provided a list of 5 criteria used for selection of custom feedback ROIs per subject: including (i) robust and typical hemodynamic response 
shown in ROI, (ii) high tSNR and tCNR, (iii) ample evidence for the ROI's involvement in the selected activation strategy, (iv) insensitivity to susceptibility artefacts, and (v) the ROI should consist of 10-15 neighbouring voxels spanning 3 fMRI slices. As post-real-time quality control, Koush et al. (2012) report the use of 4 quality metrics to evaluate their realtime denoising algorithms (tSNR, event-related tSNR, tCNR and statistical t-values), while Zilverstrand et al. (2017) used mean displacement and tSNR to investigate offline data quality differences between control and test groups. Thibault et al. (2018) suggested a list of best practices for rtfMRI-NF studies spanning the whole process from study design to outcome measurement, including suggestions for: (i) study pre-registration, (ii) sample size justification, (iii) inclusion of control neurofeedback measures, (iv) inclusion of control groups, (v) collection and reporting of the BOLD neurofeedback signal, (vi) collection and reporting of behavioural data, and (vii) outcome measure definitions and reporting.

In this work, we propose both wider adoption of such best practices in rtfMRI-NF, as well as more granular specification of data quality measurement and reporting concerning the processing steps that could influence the quality of the signal being regulated.

\subsection{Data quality challenges in rtfMRI-NF}

Real-time fMRI is plagued by the same noise fluctuations and artefacts present in conventional task-based and resting state fMRI with the main difference being the required real-time removal of these contaminants per volume, versus offline otherwise. This has to be achieved with an altered technical setup compared to the conventional approach. This timeconstrained and technically novel scenario brings about a range of challenges, discussed below.

\subsubsection{Inseparability of data measures and subject regulation effects}

A major challenge in assessing neurofeedback signal quality is the inherent mediation of the real-time signal by the process of neurofeedback training. This mediation effect, and in fact neurofeedback learnability itself, is highly variable within and between subjects and unlikely to be estimated or predicted accurately. An estimated $15-30 \%$ of subject are unable to learn control over brain computer interfaces (BCIs; Vidaurre and Blankertz, 2010), while in a review of psychological factors influencing neurofeedback learning outcomes, Kadosh and Staunton (2018) found attention, among other factors, to be crucial for neurofeedback learning success. The inability to reliably separate the rtfMRI signal into BOLD regulation effects versus noise (or noise-absent signal) makes standard quantitative measures like tSNR ill-suited to granularly assess the quality of the neurofeedback signal. Alternative measures or procedures become necessary, an example being the framework for offline evaluation and optimization of real-time neurofeedback algorithms recently put forward by Ramot and Gonzalez-Castillo (2019).

\subsubsection{Decreased statistical power}

In offline fMRI denoising, data for the whole session is available and there is effectively no time limit on the processing, which respectively allows improved statistical power for noise detection and the execution of complex algorithms to model and remove noise fluctuations. Conversely, in rtfMRI-NF the statistical power is decreased, specifically in a moving window approach or during the start of a cumulative approach due to the small amount of data samples available. Additionally, the available calculation time in real-time is limited to the span of a single TR (in the standard case of continuous feedback), albeit mostly with fewer 
data to process. This means that rtfMRI algorithms can less likely detect true BOLD effects (or noise effects) as they occur, resulting in diminishing quality control of the rtfMRI-NF signal.

\subsubsection{Lack of readily available peripheral measurements}

Most scanner setups require custom modifications to hardware and/or software in order for extra physiological information to be transferred in real-time. For example, to our knowledge few reports exist of physiological data (respiration and heart rate) being transferred and incorporated into a rtfMRI-NF software pipeline to remove physiological noise in real-time (e.g. Bodurka et al., 2009; Misaki et al., 2015; Hamilton et al., 2016). Addressing this challenge (technologically and algorithmically) could potentially be of substantial benefit to the quality of the neurofeedback signal, as it would diminish the possibility of subjects being trained on physiological nuisance signals (e.g. respiration effects) and would thus increase the contingency of the signal on actual brain activity.

\subsubsection{Difficulty of real-time visual quality control}

The neurofeedback signal is calculated and fed back to the subject immediately after the relevant preprocessing and analysis has been completed within a single TR, i.e. there is no time for an expert to inspect the volume, to assess its quality, and to perform conditional denoising steps, as opposed to offline fMRI quality control. However, this challenge provides an opportunity for rtfMRI-NF to improve computational/methods reproducibility, because a potential solution would be to have automated data quality inspection and control per volume. An example would be calculating framewise displacement (FD; Jenkinson et al., 2002; Power et al., 2012) per volume using real-time volume realignment (or head motion) parameters and automatically classifying the volume as a motion outlier or not based on some predetermined FD threshold. These outliers, in turn, could be added to a real-time motion outlier regressor in a cumulative or incremental GLM, to achieve real-time scrubbing, the results of which could be inspected and compared to offline counterparts after the rtfMRI experiment. Such functionality is currently available in rtQC (Heunis et al., 2019a)

\subsubsection{Differences in quality between real-time and offline fMRI}

Differences can occur in fMRI data that are reconstructed and transferred in real-time compared to offline exported data, including differences in voxel intensity scaling, image orientation and image header information (Hellrung et al., 2017). Such issues, if known about at all, are hardly reported by rtfMRI-NF studies, even though it could lead to potential differences of interpretation when analysing online vs. offline data. Most rtfMRI-NF studies process data offline in order to show the effects of neurofeedback training over time, often looking at the t-statistic and clustering of significantly activated voxels in a region of interest. If this analysis is carried out on different datasets because of online-offline quality control issues, conclusions could vary.

Several methods, applied during acquisition and data processing phases as well as offline, have been reported to decrease the detrimental effects of known fMRI noise and artefacts on the quality and SNR of the real-time BOLD signal. The next section investigates a set of 128 rtfMRI-NF studies to determine the prevalence of a variety of preprocessing steps in realtime fMRI pipelines, while the section thereafter focuses on the methods that address, at least in part, some of the above challenges. 


\section{Denoising in real-time fMRI neurofeedback studies}

A recent critical systematic review by Thibault et al. (2018) assessed 99 rtfMRI-NF studies in order to evaluate the effectiveness of reported experimental protocols to train subjects to selfregulate their BOLD signal and to induce behavioural improvements. The list featured a prominent set of the most recent rtfMRI-NF studies spanning a variety of patient groups and feedback signal types, and also included all 12 studies used by Emmert et al. (2016) in one of the only rtfMRI-NF meta-analyses conducted to investigate the mechanism of brain regulation resulting from neurofeedback. Apart from its main findings, the review by Thibault et al. (2018) showed that 62 out of 99 studies did not report any account being taken of respiratory confounds, that 19 studies subtracted activity in a background region to account for so-called global effects, and that 9 studies regressed out respiration-related noise signals in real-time. Respiration is known to be a source of global BOLD fluctuations and its removal is seen as a standard preprocessing step in conventional resting state fMRI processing (Bright and Murphy, 2013).

To facilitate further meta-analyses and systematic reviews, studies should not only ensure a high level of data quality (in terms of the real-time BOLD and neurofeedback signals) but also have to consistently and comprehensively report their use of acquisition and processing methods. A further search of rtfMRI-NF literature (including methods reviews) showed that rtfMRI-NF processing methodology has been covered in some detail (e.g. Weiskopf et al., 2004a; Bagarinao et al. 2006; Caria et al., 2012), but that real-time fMRI denoising methods have not received similar attention on a more granular level. To quantify the extent to which rtfMRI-NF studies report correcting for commonly known fMRI noise sources and artefacts, we investigated whether 128 recent studies (available at http://bit.ly/rtfmri-nf-zotero-library) reported the use of a standard list of real-time preprocessing steps. We conducted a Web of Science search across All Databases on 9 April 2019 using the same search terms and selection criteria as provided by Thibault et al. (2018), and found another 29 studies in addition to the original 99. The list of preprocessing steps was selected based on established practices in conventional task-based and resting state fMRI (Poldrack et al., 2011), as well as through identifying steps specific to rtfMRI-NF during the process of reviewing the 128 studies and further literature. The full text of each article, including supplementary material, were searched and coded for the following key terms: averag*, band, cutoff, difference, differential, drift, filter, frequency, heart, high, linear, low, motion, movement, nuisance, outlier, parameter, pass, physiol*, respir*, retroicor, scale, scrub, slice, smooth, spike, trend. All study DOIs and coded preprocessing steps are available as part of the accompanying Supplementary Material (Excel sheet, JSON file, Text file and Notes). Data and code necessary to reproduce Figs. 6 and 7 are available on Github (https://github.com/jsheunis/quality-and-denoising-in-rtfmri-nf).

Fig. 6 shows the list of preprocessing and denoising steps and the amount of studies that report employing these methods. Importantly, we classified studies as Do Not Report (DNR) if no mention of the particular method was made in the article or supplementary material, and if we could not confidently infer its use from studying the particular article's content. A possible exception to this rule is volume realignment, which could reasonably be expected to be used in almost all recent rtfMRI-NF studies. Fig. 6 shows that 14 out of 128 studies did not report applying volume realignment, and through investigating toolbox use (for a full distribution see Fig. 7E) it was found that the majority of these used Turbo-BrainVoyager (TBV; Brain Innovation, Maastricht, The Netherlands), which does allow including this as a standard real-time preprocessing step. Similar discrepancies could be expected in the 
classification of studies as DNR for any of the other denoising steps, although such discrepancies are expected to decline with the reported use of more non-standard or novel techniques (e.g. real-time physiological noise regression). Even so, these findings highlight the importance of correctly reporting rtfMRI-NF denoising methods, so as to promote methods reproducibility.

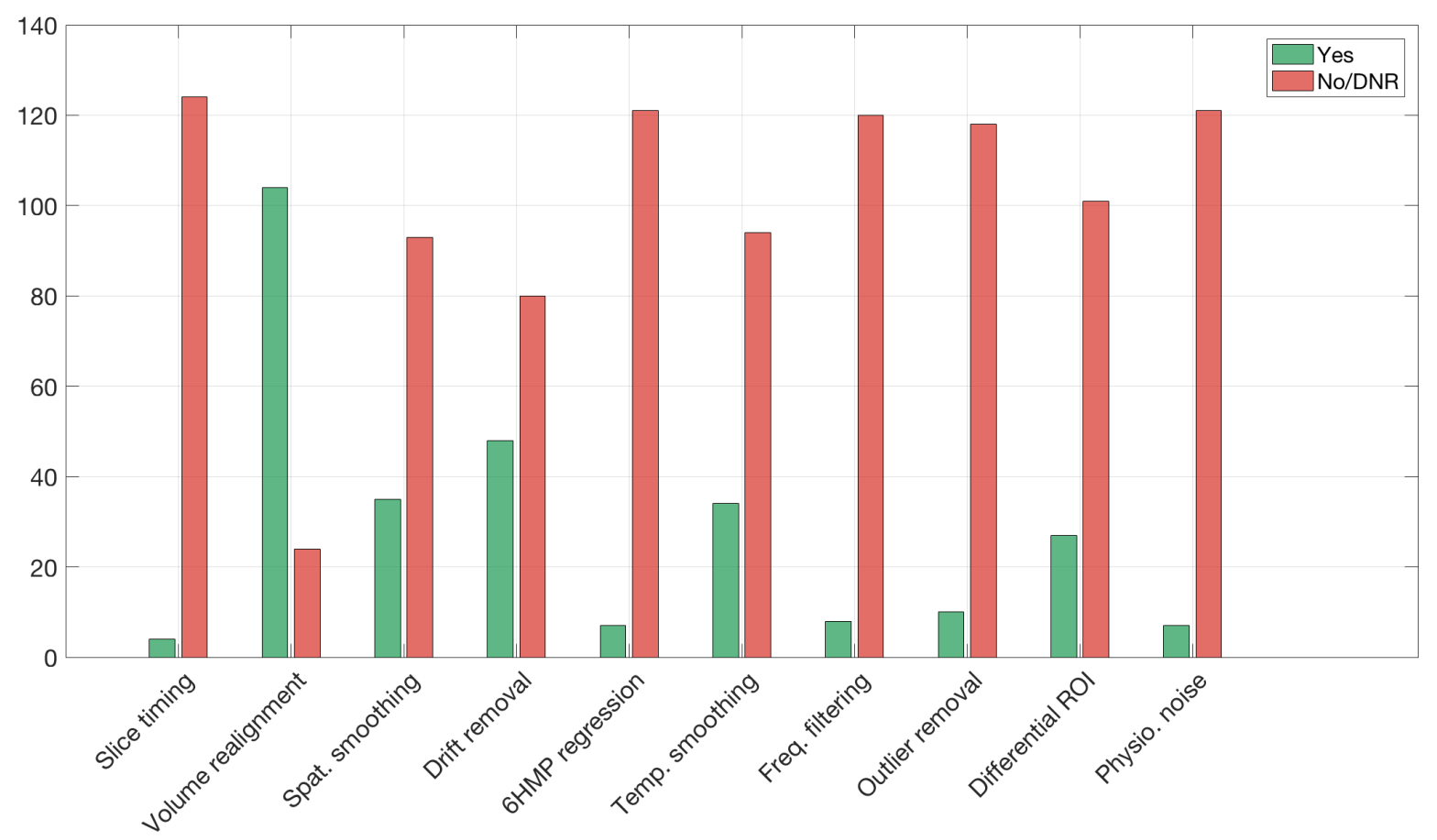

Fig. 6: A list of real-time preprocessing and denoising steps used in 128 recent rtfMRI neurofeedback studies.

Further results in Fig. 6 show that volume realignment is the only step reported to be used by over half of the studies, while less than half report implementing linear drift removal and less than a third report the use of spatial smoothing, temporal smoothing and outlier removal. A special case of real-time denoising is correction for physiological noise, where multiple approaches have been used (Fig. 7E). An often-used method is differential feedback (Weiskopf et al. 2004a; see section 5.2.8 for a full description), based on the assumption that global effects caused by respiration will be cancelled out when subtracting the averaged signal in a task-unrelated ROI from the main ROI used for neurofeedback. This also assumes that global respiration effects in both areas are identical. Still, two thirds of the studies do not report any correction for physiological noise (either in real-time or offline), while 6 studies use modeled physiological noise regression or data driven methods to remove noise fluctuations possibly caused by subject physiology (Thibault et al., 2018, reported 9 due to a mischaracterization of offline physiological noise regression as real-time regression in some cases). Additionally, it was also found that while several studies reported the use of optimized acquisition sequences to reduce susceptibility-induced image distortion (e.g. the spiral-in/out sequence by Glover and Law, 2001), only 1 study from the 128 reported incorporating postacquisition distortion correction into their real-time algorithm (Marxen et al., 2016). 


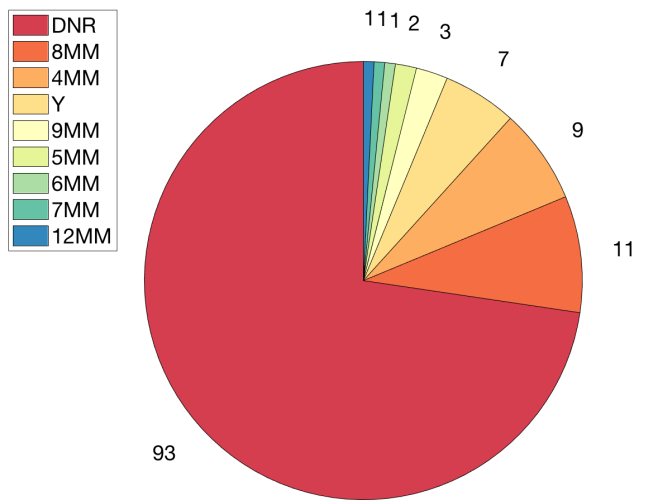

A - Spatial smoothing

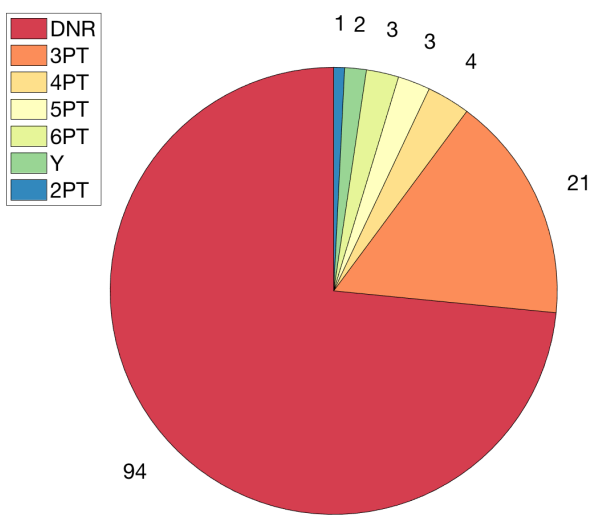

\section{C - Temporal smoothing}

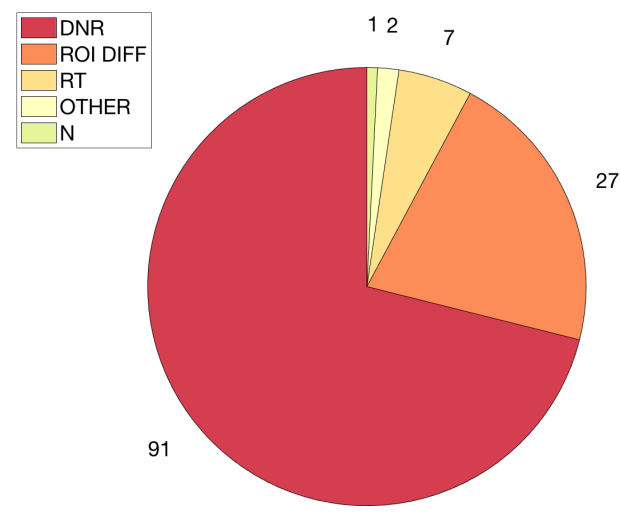

E - Respiratory noise removal

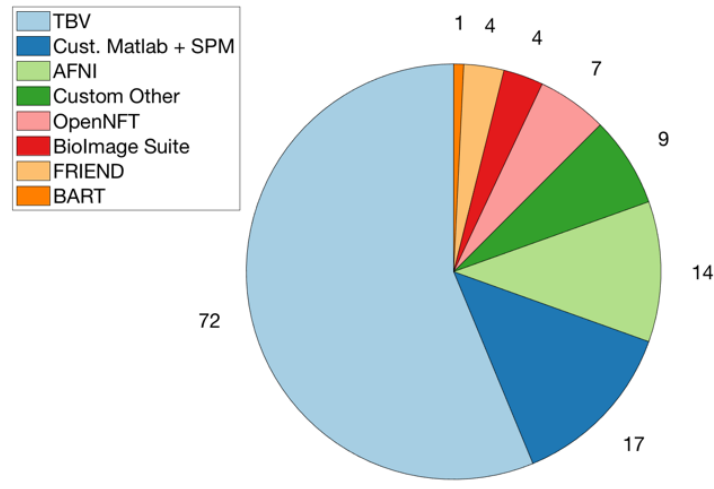

G - Real-time fMRI software

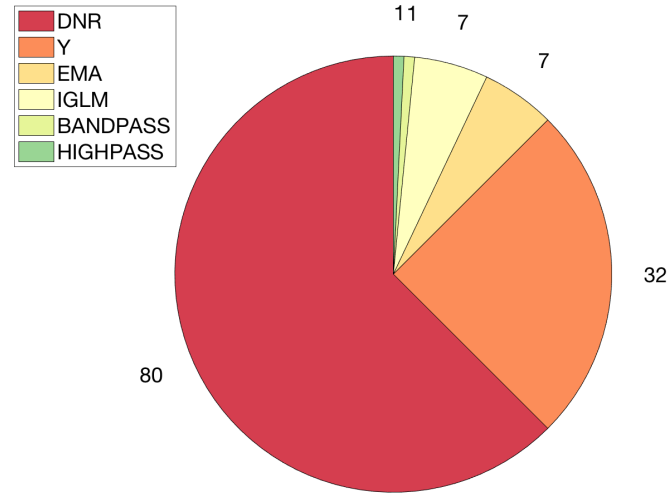

B - Drift removal

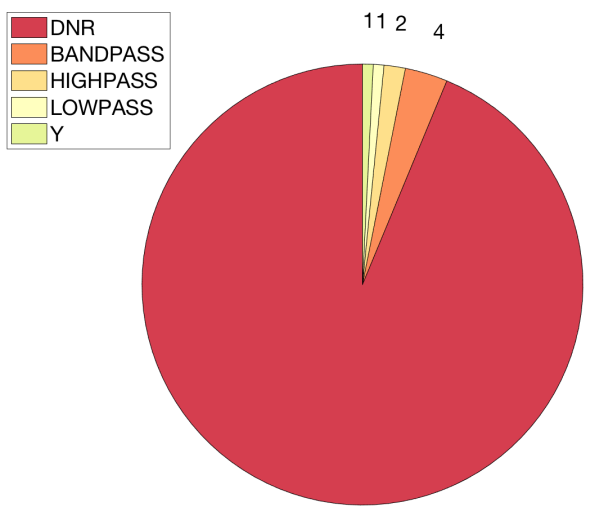

120

D - Frequency filtering

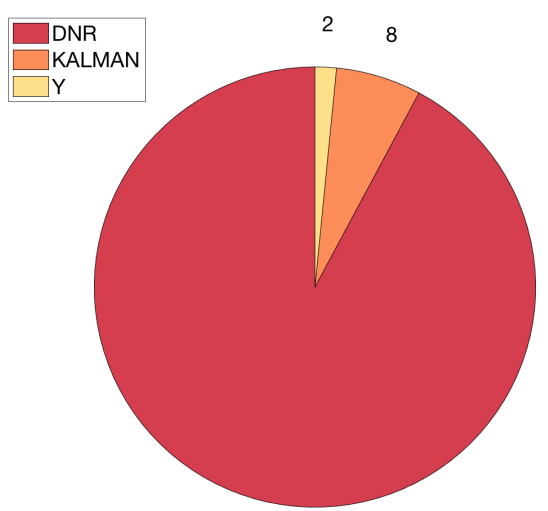

118

F - Outlier removal

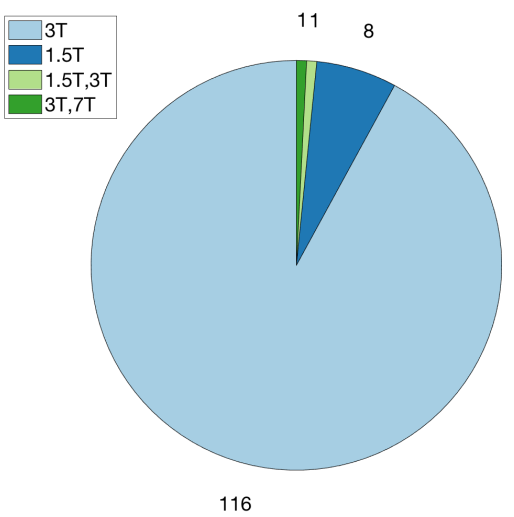

H - Magnetic field strengths 
Fig. 7. Pie charts showing a breakdown of methods used for specific preprocessing and/or denoising steps in the 128 studies compiled in this work. ( $\mathrm{DNR}=$ do not report; $\mathrm{Y}=$ yes, but no further detail reported; $\mathrm{N}=$ no):

(A) Spatial smoothing $(4,5,6,7,8,9,12 \mathrm{MM}=$ FWHM size of Gaussian smoothing kernel)

(B) Drift removal (EMA = exponential moving average filter; IGLM = incremental general linear model; BANDPASS, HIGHPASS = filter types)

(C) Temporal smoothing through time point averaging $(2,3,4,5,6 \mathrm{PT}=$ number of time points used)

(D) Frequency filtering in addition to drift removal (BANDPASS, HIGHPASS, LOWPASS = filter types)

(E) Physiological noise removal (ROI DIFF $=$ differential region of interest; RT $=$ real-time; OTHER $=$ other methods including averaged compartment signal regression, e.g. white matter and/or ventricle signals)

(F) Outlier or spike removal (KALMAN = modified Kalman filter implemented in OpenNFT)

(G) rtfMRI-NF software toolboxes (TBV = Turbo-BrainVoyager)

(H) Magnet field strengths

Finally, Thibault et al. noted a lack of both preregistration of rtfMRI-NF study designs and registered report type publications, as well as a lack of adoption of general open science principles. Although open source software solutions like AFNI's real-time plugin (Cox et al. 1995), BART (Hellrung et al., 2015), FRIEND (Basilio et al., 2015) and OpenNFT (Koush et al., 2017) counter some of these concerns, we find additionally that minimal evidence exists for open data and methods sharing. Specifically, apart from a large dataset on default-modenetwork neurofeedback shared publicly by McDonald et al. (2017), and further useful supplementary data shared in some cases (e.g. Zilverstrand et al., 2017), we found no other publicly available rtfMRI-NF datasets related to the investigated studies.

\section{Methods to improve signal quality and denoising in real- time fMRI neurofeedback}

This section addresses the third research question of this review: which methods for denoising data and improving data quality and SNR are available to the researcher studying rtfMRI$N F$ ? We consider acquisition methods and processing methods, focusing on the latter, and investigate current rtfMRI-NF algorithms and their capabilities with regards to the reported noise mitigation or denoising methods. Some offline methods for neurofeedback signal quality checking, although not strictly real-time, are also considered.

\subsection{Acquisition methods}

As with conventional fMRI, it is recommended that researchers take the necessary precautions to mitigate the introduction of any unwanted noise sources into the data. This includes the possibility of using physical interventions - e.g. individualized head restraints (https://caseforge.co/), bite bars, foam pads or end-tidal forcing systems - to counter head motion or respiratory rate variation artefacts, respectively, but also extends to tweaking pulse sequence parameters or implementing alternative sequences to increase BOLD sensitivity. Several pulse sequences, hardware changes and other acquisition steps are highlighted below.

\subsubsection{EPI, acceleration and high field imaging}

The gradient echo EPI sequence still remains the most widely used technique for real-time fMRI, as it allows fast acquisition of volumes covering the whole brain. The main disadvantages of the EPI sequence are that it is sensitive to susceptibility effects and machine instabilities, although all major vendors offer techniques that compensate (partly) for these scanner effects. 
The EPI sequence also allows for the acquisition of multiple echoes. The basic advantage of multi-echo over standard single-echo EPI is that it allows more data to fit an assumed monoexponential decay curve, which can yield voxel-wise estimations of $\mathrm{S}_{0}$ (initial signal intensity, i.e. magnetization) and $\mathrm{T}_{2}{ }^{*}$ (transverse relaxation time, Posse et al., 1998). Increased BOLD sensitivity results when these spatially varying $\mathrm{T}_{2} *$ values are combined so as to leverage optimal BOLD contrast at each voxel, as opposed to assuming a single $\mathrm{T}_{2}{ }^{*}$ for all grey matter voxels as per single-echo EPI processing. Posse et al. (1999) incorporated this advantage into their "TurboPEPSI" imaging technique for spectroscopic imaging, and later adapted it into the FIRE software toolbox for use in real-time fMRI (Posse et al., 2000), using linear echo summation. Further improvements led to the development of a method, using TurboPEPSI, for quantitative $\mathrm{T}_{2}{ }^{*}$ mapping as well as compensation of susceptibility related signal losses in multiple brain regions at different echo times (Posse et al., 2003). Later, Weiskopf et al. (2005) implemented a real-time multi-echo EPI acquisition sequence that corrected for dynamic distortions along the phase-encoding direction without the need for additional reference scans (as per standard static B0 field correction techniques).

The EPI sequence can also be combined with various accelerations techniques. The introduction of parallel imaging techniques, e.g. SENSE and GRAPPA, has contributed significantly to improved spatial resolution in fMRI, and has become standard in recent imaging applications. Also, advancements in acquisition speed using multi-band or 3D EPI techniques can improve temporal resolution as well as real-time SNR characteristics, e.g. as demonstrated by the multi-slab echo volumar imaging techniques of Posse et al. (2012) implemented in real-time.

Lastly, imaging at higher field strengths can improve SNR and BOLD sensitivity (Triantafyllou et al., 2005), both of which is beneficial to real-time fMRI neurofeedback. An important consideration for neurofeedback at $7 \mathrm{~T}$ is that physiological noise increases with field strength and may dominate the BOLD signal of interest (Krüger and Glover, 2001), necessitating an appropriate denoising procedure. If accurately accounted for, however, the increased BOLD sensitivity at $7 \mathrm{~T}$ could improve the quality of the neurofeedback signal, which would allow closer examination of the hypothesised coupling between learning effects and the neurofeedback signal. rtfMRI-NF at 7T has been implemented (e.g. Hollmann et al., 2008; Andersson, 2011) and compared to rtfMRI-NF at 3T (Gröne et al., 2015). The latter study found slightly greater increases in post-neurofeedback ROI activation in the 3T subject group compared to the 7T group. The difference was ascribed to a decrease in tSNR in the 7T group compared to the 3T group, due to several contributing factors including shimming conditions, B1-field inhomogeneities, phase-encoding polarity and physiological noise.

\subsubsection{Alternative sequences and shimming}

Alternative sequences or shimming practices have also been used to minimize the real-time image distortion or dropout artefacts related to local susceptibility gradients or other causes. A sequence developed by Glover and Law (2001) follows a spiral in/out readout trajectory of k-space that reduces signal dropout and increases BOLD contrast. Spiral-in has the advantage that it allows for higher temporal resolution, while spiral-out allows for short echo times which could also be an advantage in multi-echo denoising applications (e.g. when regressing the short echo signal out of the acquired data to remove proximal $\mathrm{S}_{0}$ effects; Bright and Murphy, 2013). Spiral in/out acquisition has been implemented by a number of rtfMRI-NF studies (Hamilton et al., 2010; Greer et al., 2014; Hamilton et al. 2016). Real-time shimming to account for geometric distortion has also been implemented. Here, Ward et al. (2002) 
implemented a sequence to detect and correct for linear shim changes in real-time, while Van Gelderen et al. (2007) used a reference B0 scan and chest motion data to apply respirationcompensating B0 shims in real-time.

\subsubsection{Prospective motion correction and motion feedback}

In addition to various MRI acquisition methods, prospective motion correction is another step that could increase SNR of the rtfMRI BOLD signal during the acquisition phase. Imagebased motion detection (Thesen et al., 2000) or external optical tracking methods (Zaitsev et al., 2006) have been used to estimate rigid body transformations and subsequently update pulse sequence parameters in real-time, such that the imaging volume essentially "follows" the subject's movement (Maclaren et al., 2012). Another method to curtail subject head motion is to feed back the head motion parameters (HMPs), derived from real-time head motion correction algorithms, to the subject. This in itself is a form of biofeedback training, and has been shown to reduce subject motion during scanning. In the case of Yang et al. (2005), head motion parameters resulting from real-time motion correction of functional image volumes were presented to subjects in the form of a composite 'head motion index', similar to framewise displacement. Greene et al. (2018) used FD as the feedback measure in their implementation using FIRMM software. Importantly, the implications of feeding back several measures to the subject and displaying them together with task instructions have to be properly understood and weighed before deciding on its use.

\subsubsection{Adaptive paradigms}

Adaptive paradigms provide interventions at a variety of stages in the acquisition and processing pipeline and allow selective data acquisition or presentation based on subjectspecific measures and a predefined set of criteria. For example, Wilms et al. (2010) developed a system where real-time eye-tracking data could be used to generate gazecontingent stimuli during fMRI experiments, while Hellrung et al. (2015) created an integrated, open-source framework for adaptive paradigm design, which also allows the dynamically updated design (based on a gaze direction-contingent assessment in their pilot experiment) to be transferred to the real-time GLM for adaptive processing. Such interventions could improve data quality and SNR by earmarking the volumes during which the subject adhered to selected quality control criteria.

\subsubsection{Peripheral data collection}

Lastly, the collection of peripheral subject data (e.g. heart rate, respiration rate, motion/eye tracking) for denoising purposes is strongly recommended. While it may not always be possible to correct for physiological noise or motion in real-time using these measures (given technical constraints or other reasons, see below) they should at least be used offline to calculate and comment on correlations with BOLD fluctuations, task design or other subjectrelated or experimental confounders.

\subsection{Processing methods}

As previously mentioned, real-time denoising methods tend to follow the course of standard offline fMRI preprocessing, although reports on the use of individual steps vary. Section 4 provided a list of real-time preprocessing or denoising steps employed by recent rtfMRI-NF studies, with Figs.6 and 7 indicating their relative usage. These methods are presented below together with other techniques identified through further literature search. 


\subsubsection{Distortion correction}

Geometric distortion effects, if addressed, are mostly accounted for using specialised acquisition methods as presented in the previous section, although correction through realtime processing is possible. An example is the point spread function (PSF) mapping approach developed by Zaitsev et al. (2004) that is used in combination with parallel imaging techniques to allow fast and fully automated distortion correction of EPI. Note that this was implemented on scanner infrastructure and not as part of an external real-time fMRI software toolbox, and that it requires a reference PSF map scan. This method was used in a rtfMRI-NF study at 3T with multi-echo EPI by Marxen et al. (2016). Another example is the dynamic, multi-echo distortion correction sequence implemented by Weiskopf et al. (2005), also implemented on scanner infrastructure.

\subsubsection{Slice timing correction}

Slice timing correction interpolates the data of different 2D slices acquired at slightly different time points along the hemodynamic response, such that the resulting 3D image represents brain activity sampled at the same time point (Sladky et al., 2011). It has been suggested that event-related analysis in fMRI is relatively robust to possible slice timing problems in sequences with a TR $\leq 2$ s (Poldrack et al., 2011). With dynamic causal modeling (DCM), which assumes a single time point sampling of all 2D slices in an fMRI volume, Kiebel et al. (2007) showed with simulations that exclusion of slice timing correction leads to larger deviations from the true connectivity parameters. They showed further that this problem is easily overcome by including slice timing correction in data preprocessing, a suggestion echoed by Koush et al. (2015) in their implementation of DCMbased neurofeedback.

Although several rtfMRI-NF toolboxes allow real-time slice timing correction (e.g. OpenNFT, Turbo-BrainVoyager), few rtfMRI-NF studies report its use, which might be explained by reporting discrepancies or by the generally short TR used in typical neurofeedback studies. To our knowledge, no studies have been conducted to determine its usefulness in rtfMRI-NF.

\subsubsection{D volume realignment}

As one of the major noise sources in fMRI, head motion received much attention during initial algorithm development in real-time fMRI. Cox and Jesmanowicz (1999) developed a fast method for 3D image registration in real-time that was incorporated into AFNI's realtime fMRI module (Cox et al. 1995), while Matthiak and Posse (2001) developed the EMOTIONAL FIRE algorithm to perform 3D rigid body realignment as part of the FIRE rtfMRI package (Gembris et al., 2000). Most other rtfMRI-NF toolboxes or custom software implementations allow some form of 3D volume realignment, e.g. OpenNFT (Koush et al., 2017) which uses a faster version of SPM12's spm_realign routine (SPM, www.fil.ion.ucl.ac.uk/spm), or FRIEND (Basilio et al., 2015) that incorporates FSL's MCFLIRT algorithm (FSL, https://fsl.fmrib.ox.ac.uk/fsl).

Regression of the 6 head motion parameter time courses (and their framewise derivatives and/or squares derived by Volterra expansion) is a typical step used in conventional fMRI preprocessing to correct for residual motion effects (Friston et al., 1996). This can be implemented in the incremental or cumulative GLMs typically used in real-time fMRI, and 
some studies have reported its use (Hamilton et al., 2016; Harmelech et al., 2013/2015; Kim et al., 2015; Yamashita et al., 2017).

\subsubsection{Spatial smoothing}

Spatial smoothing of fMRI volumes with a Gaussian kernel is typically recommended to increase the SNR for detection of signals with a spatial extent larger than a few voxels (Poldrack et al., 2011). Given that the neurofeedback signal is typically derived (per volume) from averaging the signal intensity over multiple voxels within an ROI, a basic form of spatial smoothing is inherently applied. It could be argued that this negates the need for an extra spatial smoothing step in the real-time fMRI processing pipeline, but further research is necessary to determine this argument's validity. Numerous rtfMRI-NF studies report spatially smoothing their fMRI data before calculating the neurofeedback signal, while in some cases (e.g. neurofeedback based on MPVA of voxels within an ROI) it might be explicitly excluded.

\subsubsection{Linear detrending / drift removal}

Correcting for signal drift is a relatively standard step in real-time fMRI and could form part of the real-time GLM procedure, where a linear term and/or basis set of low frequency drift terms are included as regressors, acting as a high-pass filter. An inherent correction for baseline drift is also executed in some percentage signal change neurofeedback paradigms during feedback signal calculation, due to the cumulative global mean being subtracted from the averaged ROI BOLD signal (e.g. deCharms et al., 2005; Garrison et al., 2013). Most major rtfMRI-NF toolboxes allow some form of low-frequency drift correction. In a recent study, Kopel et al. (2019) compared the performance of commonly used online detrending algorithms with regards to their ability to eliminate drift components and artefacts without distorting the signal of interest. They found performance to be similar for exponential moving average (EMA), incremental general linear model (iGLM) and sliding window iGLM $\left(\mathrm{iGLM}^{\text {window }}\right)$, although the latter option was proposed for future studies.

\subsubsection{Temporal filtering or averaging}

Further filtering of real-time fMRI data is possible, for example with the exponential moving average filter employed by Koush et al. (2012) to remove both high frequency noise and low frequency drift from the BOLD signal, or by including regressors relating to a specific frequency pass-band in the real-time GLM. Averaging of timepoints before calculating the neurofeedback signal, using a moving window approach, is another step implemented in several rtfMRI-NF studies (e.g. Young et al., 2014).

\subsubsection{Outlier or spike removal}

Removal or replacement of outlier volumes or data based on some quality criteria (whether defined visually or according to data calculations) is a method employed in conventional fMRI analysis to improve SNR (Power et al., 2014). Similar steps have been taken in realtime fMRI, for example in the BioImage Suite and custom Matlab implementation of Garrison et al. (2013), where a volume is classified as an outlier and replaced by the previous volume if mean activation in the ROI differed by more than $10 \%$ from the previous measurement. Koush et al. (2012) implemented an adapted Kalman filter, by applying nonlinear modifications, that define outliers by their irregular statistical properties in order to achieve spike detection and high frequency filtering. This algorithm has been incorporated into the open-source OpenNFT toolbox as part of its standard real-time processing pipeline 
(Koush et al., 2017). Additionally, the Kalman filter requires only the current datapoint and previous state information, as opposed to all previous data points (or a subset thereof), and therefore does not add much latency to the real-time pipeline. Lastly, outlier rejection based on a standardized voxel intensity threshold has also been reported by McCaig et al., (2011), in which they exclude voxels with a standardized intensity of $z<-2.0$ from the real-time ROI analysis in order to reduce noise associated with out-of-brain voxels and signal dropout.

\subsubsection{Accounting for global effects through differential feedback}

Feedback on the difference signal between ROIs has been motivated as a way to cancel out global effects like global intensity changes caused by respiration-induced artefacts (Weiskopf et al., 2004a, 2004b; deCharms et al., 2004). In addition to the main ROI selected for neurofeedback, a reference or background ROI is typically defined as a task-unrelated axial slice or 3D ROI, in which the average signal is calculated and subtracted from the main ROI. Alternatively, defining the reference ROI as another task-related region allows subjects to attempt more specific bidirectional control of brain activity due to general regulation effects being cancelled out, for example using both the supplementary motor area and the parahippocampal place area as ROIs for PSCNef (Weiskopf et al., 2004b). These points have motivated several studies to opt for differential feedback over standard (non-differential) feedback, although a limitation would be that global effects may in fact vary substantially across the brain and that differential feedback might actually decrease SNR if activation related information is contained within the reference ROI (Marins et al., 2015). To our knowledge, no experiments have been conducted and published that investigate the relationship between differential feedback and SNR of the feedback signal, thus further research would benefit this area.

\subsubsection{Physiological noise correction (respiration and heart rate)}

Denoising physiological confounds has been approached in a variety of ways in rtfMRI-NF, even though most studies do not report any correction for physiological noise. In those that do, differential feedback is most often used as a potential correction method for global effects caused by respiration (although accompanied by above mentioned caveats). Filtering can also remove some physiology-induced variance, with the modified Kalman filter by Koush et al. $(2012,2017)$ being a special case where high-frequency spikes resulting from changes in head position or breathing can be filtered out with no prior assumption about the specific noise model. Another option to remove physiology-related variance is to regress the spatialaveraged time course of compartments like white matter or the ventricles from the signal of interest, i.e. a real-time version of tissue-based nuisance regression as conventionally used in offline analyses. Spetter et al. (2017) calculated partial correlation of areas of interest with white matter and used these results to regress out any unwanted fluctuations before the neurofeedback signal was calculated, and Yamashita et al. (2017) included averaged signals from white matter, grey matter and CSF as nuisance signals in their real-time regression analysis.

Model-based approaches follow the work done by Glover et al. (2000), Birn et al. (2006) and Chang et al. (2009) on retrospective image correction (RETROICOR), respiratory volume per time (RVT) and heart rate variability (HRV), respectively, where concurrent recordings of the subject's breathing and heart rate are used to create nuisance regressors used in subsequent real-time linear modeling. With physiological signal monitoring built into AFNI's real-time plugin (Bodurka et al., 2009), Misaki et al. (2015) implemented the first real-time RETROICOR and RVT physiological regression as an extension, using a GPU to denoise 
over $100 \mathrm{k}$ voxels (i.e. whole brain data) in under $300 \mathrm{~ms}$ per volume. Hamilton et al. (2016) reported including two physiological noise regressors in their real-time regression analysis implemented in custom $\mathrm{C} / \mathrm{C}++$ and Matlab, with no further detail provided.

Time synchronization of peripheral recordings and fMRI data is a legitimate challenge to model-based correction of breathing and heart rate variability artefacts in real-time, unless the challenge is avoided altogether by using advanced processing power and full recalculation of all available data for every iteration, as was done by Misaki et al. (2015). Some global timestamping solutions have been implemented to allow synchronization of concurrent physiology and fMRI recordings (Smyser et al., 2001; Voyvodic et al., 2011; Hellrung et al., 2015). This typically requires a custom-programmed software package dedicated to managing time-synchronisation of multiple concurrent inputs and outputs, for example the BART CIGAL software (Voyvodic et al., 1999) which could run modules in parallel for the main stimulus event, a button-press hardware input, an analog data input for physiological recordings, the scanner trigger, eye-tracker recordings of eye position and pupil diameter, and more.

Lastly, we found no examples of studies investigating and comparing the efficacy of different physiological noise removal strategies or their effect on the neurofeedback signal in rtfMRINF.

\subsubsection{Other real-time processing methods}

Global signal regression, although a controversial denoising step in offline fMRI processing (Murphy and Fox, 2017), can be used in real-time to remove global fluctuations common to large areas of the brain and hypothesized to be of non-neuronal origin. This would typically involve including the cumulative global mean signal in the real-time GLM and regressing that out of the averaged ROI BOLD signal of interest, similar to CSF and white matter compartment regression.

Independent component analysis (ICA) has been a very effective tool in finding nuisance networks in resting state fMRI, which can be regressed out of the fMRI time series for effective denoising. Esposito et al. (2003) were the first to implement a real-time ICA algorithm using a sliding-window approach on a limited amount of axial brain slices, as a plugin to Turbo-BrainVoyager. Although this was used to generate quasistationary activation maps and accompanying time courses, this demonstration sufficed to highlight the possibility of generating the spatiotemporal characteristics of nuisance signals for real-time denoising. This functionality, however, has not extended towards wider exploration or adoption.

Voxel efficiency scaling was proposed and implemented by Hinds et al. (2011) in their software toolbox Murfi as a way to avoid the undesired noise weighting resulting from standard direct averaging of all voxels within the neurofeedback region of interest. Rather, a z-score weighted average of the ROI voxels were used for neurofeedback signal calculation, which they found to result in increased SNR of the neurofeedback signal compared to a posthoc calculation method as well as the standard direct averaging method.

Lastly, multi-echo EPI processing methods in real-time have also shown promise in increasing the SNR of the real-time BOLD signal, specifically in areas of the brain where the local $\mathrm{T}_{2} *$ is not close to the standard EPI echo time of $\sim 30 \mathrm{~ms}$ selected for optimal BOLD contrast at 3T. The multi-echo acquisition methods reviewed earlier are typically 
accompanied by echo summation schemes that allow real-time increases in BOLD sensitivity. Posse et al. (2003) implemented a fixed, linear, TE-weighted summation of echo signals, a processing scheme later also used by Marxen et al. (2016) in their neurofeedback study of the amygdala. After multi-echo image acquisition and real-time distortion correction of all echo images, Weiskopff et al. (2005) used a BOLD sensitivity curve for weighted combination. Several other combination schemes are possible (e.g. Poser et al., 2006), and in related work we have investigated the comparative performance of various real-time combination schemes in terms of tSNR distributions (Heunis et al., 2019b). Further work is necessary to determine their comparative efficacy in terms of extended quality metrics important to rtfMRI-NF.

\subsubsection{Further quality control of the feedback signal}

Some methods do not consist of efforts to denoise the real-time BOLD signal of specific nuisance fluctuations, but rather to improve the quality of data acquisition or feedback presentation in real-time. Offline methods are also used as post-hoc data quality checks.

Temporally averaging and scaling the feedback signal are often used to prevent abrupt changes to the signal presented to the subject in real-time. For example, Garrison et al. (2013) used a sliding window of 5 volumes for temporal smoothing of the mean ROI activation intended for neurofeedback. OpenNFT (Koush et al., 2017) uses a dynamic range, defined by the average of the 5\% highest and lowest acquired activity time points, to scale the dynamic feedback signal.

Lastly, several quality control methods have been proposed to determine whether respiration or heart rate fluctuations may have had any significant effect on the neurofeedback signal calculation that could bias the data. These should be separated from real-time denoising algorithms which aim to remove the noise/artefact before the feedback signal is calculated and displayed to the subject. For example, Sorger et al. (2016) collect real-time cardiac and respiratory traces and analyse them after the neurofeedback session to investigate possible correlations with the task design or other BOLD fluctuations. Physiological traces can also be incorporated into offline physiological denoising (e.g. RETROICOR) denoising when assessing the BOLD signal for neurofeedback-induced changes over time (e.g. Sulzer et al., 2013b).

\section{Discussion, recommendations and future perspective}

In this work, our goal was to shed light on the status of data quality challenges, denoising practices and methods reporting in the field of real-time fMRI neurofeedback. Prior studies in conventional fMRI have shown the implications of not sufficiently removing noise signals or not accounting for confounding effects (e.g. Gitelman et al., 2003; Van Dijk et al., 2011; Power et al., 2012; Rangaprakash et al., 2018). We aimed to investigate this in the domain of real-time fMRI neurofeedback and present our findings such that researchers can be thoroughly informed about the quality of their neurofeedback signal of interest. The aim is to assist researchers in designing rtfMRI-NF studies that avoid (as far as possible) sham learning and, subsequently, incorrect inferences.

\section{Existing denoising methods: acquisition and processing}

Literature showed that methods development during the past two decades has delivered multiple acquisition and processing methods to the researcher conducting a rtfMRI-NF study, 
implemented in the form of custom sequences and tools including Turbo-BrainVoyager, AFNI, OpenNFT, FRIEND and BART. For acquisition real-time shimming, spiral-in/out and multi-echo EPI (including multi-band) approaches show promise in reducing susceptibility induced geometric distortion and increasing BOLD sensitivity, and are recommended for future implementation. From a processing perspective, real-time denoising pipelines showed high similarity to offline counterparts, although some tradeoffs are made because of the time limitation and the iterative nature of real-time processing. The effects of inclusion or exclusion of specific denoising steps in the real-time pipeline on the quality of the neurofeedback signal were found to be unexplored except for a single study (Kopel et al., 2019). We therefore cannot make further empirically supported recommendations for specific denoising pipelines, apart from a general recommendation to account for head motion, drift, physiological noise, outliers and residual effects. This highlights the need for new methodological studies to quantify denoising step effects and compare pipelines. Collection of peripheral physiological data (e.g. heart rate, respiration rate, eye movements) is always recommended when possible, either to be used for real-time denoising or otherwise to rule out as confounds during offline analysis.

\section{Quality control in real-time fMRI neurofeedback}

While above mentioned tools and methods exist, quality control and best practices in rtfMRINF is markedly unexplored and unreported compared to conventional fMRI, where initiatives like MRIQC (Esteban et al., 2017), QAP (Processed Connectome Project, 2014), COBIDAS (Nichols et al., 2017) support improved quality control and methods reproducibility. Although some studies report the use of best practices and data quality metrics to assess their neurofeedback signal (Koush et al., 2012; Stoeckel et al., 2014; Sorger et al., 2016; Zilverstrand et al., 2017), it is unreported in the majority of the literature. Furthermore, other potential data quality issues like differences between offline and real-time acquired data, or geometric distortion unaccounted for during acquisition or real-time processing, could further skew the data, yet they remain unreported. It is our perspective that a concerted effort is necessary to establish a practical set of rtfMRI-NF quality metrics and methods that allow their calculation, visualization, comparison and reporting. This could expand on the work proposed by Stoeckel et al. (2014) and Thibault et al. (2018).

\section{Methods reporting and best practice adoption}

In our analysis of 128 recent rtfMRI-NF studies we found that less than a third of the studies reported implementing several processing steps, including slice timing correction, spatial smoothing, regression of head motion parameters, temporal averaging or filtering, outlier or spike removal, using a differential ROI to account for global effects, and further physiological noise correction. If correctly reported, this low percentage of studies raises concern about the general quality of the real-time fMRI neurofeedback signal. Researchers should report accurately on acquisition, processing and any other steps taken to remove noise fluctuations from and improve the quality of real-time fMRI and the neurofeedback signal. As a starting point, studies could include a checklist reporting the implementation of the realtime processing steps listed in this work. We have compiled a template checklist to act as a guideline, and acknowledge that this is not an exhaustive list but one that could mature over time with community input. It was compiled in the vein of the COBIDAS best practice effort and would best be interpreted as an addition to the COBIDAS reporting guidelines for realtime fMRI. An online version of this checklist is available on the Open Science Framework (https://osf.io/kjwhf/). 


\section{Future perspective}

Moving towards a scenario where the hypothesized usefulness of rtfMRI-NF in a clinical environment can be investigated and demonstrated transparently will require studies with reproducible methods and results. In light of this, we echo the recommendations made by Thibault et al. (2018) regarding reproducible science. Where possible, rtfMRI-NF studies with a clear hypothesis should be pre-registered or follow a registered report submission process. Additionally, the continued use and development of open source software solutions based on widely used neuroimage processing tools, like OpenNFT (SPM), FRIEND (FSL) or AFNI's real-time plugin, are recommended together with data sharing on platforms like OpenNeuro (https://openneuro.org/). In this way, both published data and methods can be queried by multiple researchers, paving the way for reproducible methods, results and inferences.

\section{Acknowledgements}

This work was supported by funding from the foundation LSH-TKI (grant LSHM16053-

SGF) and Philips Research. The authors are grateful to the Dutch Epilepsy foundation and in particular to Dr. Martin Boer for their support in the materialisation of this work.

\section{Author contributions}

$\mathrm{SH}, \mathrm{RL}, \mathrm{SZ}, \mathrm{BA}$ and MB conceived the manuscript outline. SH conducted the research and wrote the manuscript. RL contributed to real-time fMRI acquisition sections through discussions and writing. All authors reviewed and revised the manuscript.

\section{Conflicts of interest}

RL and MB are, respectively, employees of Philips Research and Philips Healthcare in The Netherlands. The other authors have declared that no further competing interests exist.

\section{Data availability statement}

The data, code and supplementary material that support the findings of this study are openly available. All supplementary material accompanying this manuscript can be found at: https://dx.doi.org/10.17605/OSF.IO/2N5P3. The 128 studies forming the basis of analyses in this manuscript are listed at: http://bit.ly/rtfmri-nf-zotero-library. The data and code necessary to reproduce figures in this manuscript can be found at: https://github.com/jsheunis/qualityand-denoising-in-rtfmri-nf. 


\section{References}

Alegria, A.A., Wulff, M., Brinson, H., Barker, G.J., Norman, L.J., Brandeis, D., Stahl, D., David, A.S., Taylor, E., Giampietro, V., Rubia, K., 2017. Real-time fMRI neurofeedback in adolescents with attention deficit hyperactivity disorder. Human Brain Mapping 38, 3190-3209. https://doi.org/10.1002/hbm.23584

Andersson, P., Pluim, J.P.W., Siero, J.C.W., Klein, S., Viergever, M.A., Ramsey, N.F., 2011. Real-Time Decoding of Brain Responses to Visuospatial Attention Using 7T fMRI. PLOS ONE 6, e27638. https://doi.org/10.1371/journal.pone.0027638

Bagarinao, E., Matsuo, K., Nakai, T., Sato, S., 2003. Estimation of general linear model coefficients for real-time application. NeuroImage 19, 422-429. https://doi.org/10.1016/S1053-8119(03)00081-8

Bagarinao, E., Nakai, T., Tanaka, Y., 2006. Real-time functional MRI: development and emerging applications. Magn Reson Med Sci 5, 157-165.

Balteau, E., Hutton, C., Weiskopf, N., 2010. Improved shimming for fMRI specifically optimizing the local BOLD sensitivity. Neuroimage 49, 327-336. https://doi.org/10.1016/j.neuroimage.2009.08.010

Bandettini, P.A., Jesmanowicz, A., Wong, E.C., Hyde, J.S., 1993. Processing strategies for time-course data sets in functional mri of the human brain. Magnetic Resonance in Medicine 30, 161-173. https://doi.org/10.1002/mrm.1910300204

Basilio, R., Garrido, G.J., Sato, J.R., Hoefle, S., Melo, B.R.P., Pamplona, F.A., Zahn, R., Moll, J., 2015. FRIEND Engine Framework: a real time neurofeedback client-server system for neuroimaging studies. Front Behav Neurosci 9. https://doi.org/10.3389/fnbeh.2015.00003

Bellgowan, P.S.F., Bandettini, P.A., van Gelderen, P., Martin, A., Bodurka, J., 2006. Improved BOLD detection in the medial temporal region using parallel imaging and voxel volume reduction. NeuroImage 29, 1244-1251. https://doi.org/10.1016/j.neuroimage.2005.08.042

Binder, J.R., 2011. Functional MRI is a valid noninvasive alternative to Wada testing. Epilepsy \& Behavior 20, $214-222$. https://doi.org/10.1016/j.yebeh.2010.08.004

Birn, R.M., Diamond, J.B., Smith, M.A., Bandettini, P.A., 2006. Separating respiratory-variation-related fluctuations from neuronal-activity-related fluctuations in fMRI. NeuroImage 31, 1536-1548. https://doi.org/10.1016/j.neuroimage.2006.02.048

Biswal, B., Yetkin, F.Z., Haughton, V.M., Hyde, J.S., 1995. Functional connectivity in the motor cortex of resting human brain using echo-planar mri. Magnetic Resonance in Medicine 34, 537-541. https://doi.org/10.1002/mrm.1910340409

Bodurka, J., Gonzales-Castillo, J., Bandettini, P.A., 2009. The Use of Neurofeedback with Real-Time Functional MRI to Suppress Physiological Noise. NeuroImage 47, S194. https://doi.org/10.1016/S1053-8119(09)72192-5

Bodurka, J., Ye, F., Petridou, N., Murphy, K., Bandettini, P.A., 2007. Mapping the MRI Voxel Volume in Which Thermal Noise Matches Physiological Noise-Implications for fMRI. Neuroimage 34, 542-549. https://doi.org/10.1016/j.neuroimage.2006.09.039

Bollmann, S., Kasper, L., Vannesjo, S.J., Diaconescu, A.O., Dietrich, B.E., Gross, S., Stephan, K.E., Pruessmann, K.P., 2017. Analysis and correction of field fluctuations in fMRI data using field monitoring. NeuroImage, Cleaning up the fMRI time series: Mitigating noise with advanced acquisition and correction strategies 154, 92-105. https://doi.org/10.1016/j.neuroimage.2017.01.014

Bright, M.G., Murphy, K., 2013. Removing motion and physiological artifacts from intrinsic BOLD fluctuations using short echo data. Neuroimage 64, 526-537. https://doi.org/10.1016/j.neuroimage.2012.09.043

Caballero-Gaudes, C., Reynolds, R.C., 2017. Methods for cleaning the BOLD fMRI signal. NeuroImage, Cleaning up the fMRI time series: Mitigating noise with advanced acquisition and correction strategies 154, 128-149. https://doi.org/10.1016/j.neuroimage.2016.12.018

Canterberry, M., Hanlon, C.A., Hartwell, K.J., Li, X., Owens, M., LeMatty, T., Prisciandaro, J.J., Borckardt, J., Saladin, M.E., Brady, K.T., George, M.S., 2013. Sustained Reduction of Nicotine Craving With Real-Time Neurofeedback: Exploring the Role of Severity of Dependence. Nicotine Tob Res 15, 2120-2124. https://doi.org/10.1093/ntr/ntt122

Caria, A., Sitaram, R., Birbaumer, N., 2012. Real-Time fMRI: A Tool for Local Brain Regulation. Neuroscientist 18, 487501. https://doi.org/10.1177/1073858411407205

Chang, C., Cunningham, J.P., Glover, G.H., 2009. Influence of heart rate on the BOLD signal: The cardiac response function. NeuroImage 44, 857-869. https://doi.org/10.1016/j.neuroimage.2008.09.029

Ciric, R., Wolf, D.H., Power, J.D., Roalf, D.R., Baum, G.L., Ruparel, K., Shinohara, R.T., Elliott, M.A., Eickhoff, S.B., Davatzikos, C., Gur, R.C., Gur, R.E., Bassett, D.S., Satterthwaite, T.D., 2017. Benchmarking of participant-level confound regression strategies for the control of motion artifact in studies of functional connectivity. NeuroImage, Cleaning up the fMRI time series: Mitigating noise with advanced acquisition and correction strategies 154, $174-187$. https://doi.org/10.1016/j.neuroimage.2017.03.020 
Cohen, J.D., Daw, N., Engelhardt, B., Hasson, U., Li, K., Niv, Y., Norman, K.A., Pillow, J., Ramadge, P.J., Turk-Browne, N.B., Willke, T.L., 2017. Computational approaches to fMRI analysis. Nat Neurosci 20, 304-313. https://doi.org/10.1038/nn.4499

Cox, R.W., Jesmanowicz, A., 1999. Real-time 3D image registration for functional MRI. Magnetic Resonance in Medicine 42, 1014-1018. https://doi.org/10.1002/(SICI)1522-2594(199912)42:6<1014::AID-MRM4>3.0.CO;2-F

Cox, R.W., Jesmanowicz, A., Hyde, J.S., 1995. Real-Time Functional Magnetic Resonance Imaging. Magnetic Resonance in Medicine 33, 230-236. https://doi.org/10.1002/mrm.1910330213

deCharms, R.C., 2007. Reading and controlling human brain activation using real-time functional magnetic resonance imaging. Trends in Cognitive Sciences 11, 473-481. https://doi.org/10.1016/j.tics.2007.08.014

deCharms, R.C., Christoff, K., Glover, G.H., Pauly, J.M., Whitfield, S., Gabrieli, J.D.E., 2004. Learned regulation of spatially localized brain activation using real-time fMRI. NeuroImage 21, 436-443. https://doi.org/10.1016/j.neuroimage.2003.08.041

deCharms, R.C., Maeda, F., Glover, G.H., Ludlow, D., Pauly, J.M., Soneji, D., Gabrieli, J.D.E., Mackey, S.C., 2005. Control over brain activation and pain learned by using real-time functional MRI. PNAS 102, 18626-18631. https://doi.org/10.1073/pnas.0505210102

Deichmann, R., Josephs, O., Hutton, C., Corfield, D.R., Turner, R., 2002. Compensation of Susceptibility-Induced BOLD Sensitivity Losses in Echo-Planar fMRI Imaging. NeuroImage 15, 120-135. https://doi.org/10.1006/nimg.2001.0985

Diedrichsen, J., Shadmehr, R., 2005. Detecting and adjusting for artifacts in fMRI time series data. NeuroImage 27, 624634. https://doi.org/10.1016/j.neuroimage.2005.04.039

Dosenbach, N.U.F., Koller, J.M., Earl, E.A., Miranda-Dominguez, O., Klein, R.L., Van, A.N., Snyder, A.Z., Nagel, B.J., Nigg, J.T., Nguyen, A.L., Wesevich, V., Greene, D.J., Fair, D.A., 2017. Real-time motion analytics during brain MRI improve data quality and reduce costs. NeuroImage 161, 80-93. https://doi.org/10.1016/j.neuroimage.2017.08.025

Eklund, A., Andersson, M., Knutsson, H., 2012. fMRI analysis on the GPU-Possibilities and challenges. Computer Methods and Programs in Biomedicine 105, 145-161. https://doi.org/10.1016/j.cmpb.2011.07.007

Emmert, K., Kopel, R., Koush, Y., Maire, R., Senn, P., Van De Ville, D., Haller, S., 2017. Continuous vs. intermittent neurofeedback to regulate auditory cortex activity of tinnitus patients using real-time fMRI - A pilot study. NeuroImage: Clinical 14, 97-104. https://doi.org/10.1016/j.nicl.2016.12.023

Emmert, K., Kopel, R., Sulzer, J., Brühl, A.B., Berman, B.D., Linden, D.E.J., Horovitz, S.G., Breimhorst, M., Caria, A., Frank, S., Johnston, S., Long, Z., Paret, C., Robineau, F., Veit, R., Bartsch, A., Beckmann, C.F., Van De Ville, D., Haller, S., 2016. Meta-analysis of real-time fMRI neurofeedback studies using individual participant data: How is brain regulation mediated? NeuroImage 124, 806-812. https://doi.org/10.1016/j.neuroimage.2015.09.042

Esposito, F., Seifritz, E., Formisano, E., Morrone, R., Scarabino, T., Tedeschi, G., Cirillo, S., Goebel, R., Di Salle, F., 2003. Real-time independent component analysis of fMRI time-series. NeuroImage 20, 2209-2224. https://doi.org/10.1016/j.neuroimage.2003.08.012

Esteban, O., Birman, D., Schaer, M., Koyejo, O.O., Poldrack, R.A., Gorgolewski, K.J., 2017. MRIQC: Advancing the automatic prediction of image quality in MRI from unseen sites. PLOS ONE 12, e0184661. https://doi.org/10.1371/journal.pone.0184661

Esteban, O., Markiewicz, C., Blair, R.W., Moodie, C., Isik, A.I., Aliaga, A.E., Kent, J., Goncalves, M., DuPre, E., Snyder, M., Oya, H., Ghosh, S., Wright, J., Durnez, J., Poldrack, R., Gorgolewski, K.J., 2018. FMRIPrep: a robust preprocessing pipeline for functional MRI. bioRxiv 306951. https://doi.org/10.1101/306951

Foerster, B.U., Tomasi, D., Caparelli, E.C., 2005. Magnetic field shift due to mechanical vibration in functional magnetic resonance imaging. Magnetic Resonance in Medicine 54, 1261-1267. https://doi.org/10.1002/mrm.20695

Friston, K.J., Williams, S., Howard, R., Frackowiak, R.S.J., Turner, R., 1996. Movement-Related effects in fMRI timeseries. Magnetic Resonance in Medicine 35, 346-355. https://doi.org/10.1002/mrm. 1910350312

Garrison, K.A., Scheinost, D., Worhunsky, P.D., Elwafi, H.M., Thornhill, T.A., Thompson, E., Saron, C., Desbordes, G., Kober, H., Hampson, M., Gray, J.R., Constable, R.T., Papademetris, X., Brewer, J.A., 2013. Real-time fMRI links subjective experience with brain activity during focused attention. NeuroImage 81, 110-118. https://doi.org/10.1016/j.neuroimage.2013.05.030

Geissler, A., Gartus, A., Foki, T., Tahamtan, A.R., Beisteiner, R., Barth, M., 2007. Contrast-to-noise ratio (CNR) as a quality parameter in fMRI. Journal of Magnetic Resonance Imaging 25, 1263-1270. https://doi.org/10.1002/jmri.20935

Gelderen, P. van, Zwart, J.A. de, Starewicz, P., Hinks, R.S., Duyn, J.H., 2007. Real-time shimming to compensate for respiration-induced B0 fluctuations. Magnetic Resonance in Medicine 57, 362-368. https://doi.org/10.1002/mrm.21136

Gembris, D., Taylor, J.G., Schor, S., Frings, W., Suter, D., Posse, S., 2000. Functional magnetic resonance imaging in real time (FIRE): Sliding-window correlation analysis and reference-vector optimization. Magnetic Resonance in Medicine 43, 259-268. https://doi.org/10.1002/(SICI)1522-2594(200002)43:2<259::AID-MRM13>3.0.CO;2-P 
Gitelman, D.R., Penny, W.D., Ashburner, J., Friston, K.J., 2003. Modeling regional and psychophysiologic interactions in fMRI: the importance of hemodynamic deconvolution. NeuroImage 19, 200-207. https://doi.org/10.1016/S1053$\underline{8119(03) 00058-2}$

Glover, G.H., Law, C.S., 2001. Spiral-in/out BOLD fMRI for increased SNR and reduced susceptibility artifacts. Magnetic Resonance in Medicine 46, 515-522. https://doi.org/10.1002/mrm.1222

Glover, G.H., Li, T.-Q., Ress, D., 2000. Image-based method for retrospective correction of physiological motion effects in fMRI: RETROICOR. Magnetic Resonance in Medicine 44, 162-167. https://doi.org/10.1002/15222594(200007)44:1<162::AID-MRM23>3.0.CO;2-E

Goodman, S.N., Fanelli, D., Ioannidis, J.P.A., 2016. What does research reproducibility mean? Science Translational Medicine 8, 341ps12-341ps12. https://doi.org/10.1126/scitranslmed.aaf5027

Gorno-Tempini, M.L., Hutton, C., Josephs, O., Deichmann, R., Price, C., Turner, R., 2002. Echo Time Dependence of BOLD Contrast and Susceptibility Artifacts. NeuroImage 15, 136-142. https://doi.org/10.1006/nimg.2001.0967

Green, M.V., Seidel, J., Stein, S.D., Tedder, T.E., Kempner, K.M., Kertzman, C., Zeffiro, T.A., 1994. Head movement in normal subjects during simulated PET brain imaging with and without head restraint. J. Nucl. Med. 35, 1538-1546.

Greene, D.J., Koller, J.M., Hampton, J.M., Wesevich, V., Van, A.N., Nguyen, A.L., Hoyt, C.R., McIntyre, L., Earl, E.A., Klein, R.L., Shimony, J.S., Petersen, S.E., Schlaggar, B.L., Fair, D.A., Dosenbach, N.U.F., 2018. Behavioral interventions for reducing head motion during MRI scans in children. NeuroImage 171, 234-245. https://doi.org/10.1016/j.neuroimage.2018.01.023

Greer, S.M., Trujillo, A.J., Glover, G.H., Knutson, B., 2014. Control of nucleus accumbens activity with neurofeedback. NeuroImage 96, 237-244. https://doi.org/10.1016/j.neuroimage.2014.03.073

Griffanti, L., Salimi-Khorshidi, G., Beckmann, C.F., Auerbach, E.J., Douaud, G., Sexton, C.E., Zsoldos, E., Ebmeier, K.P., Filippini, N., Mackay, C.E., Moeller, S., Xu, J., Yacoub, E., Baselli, G., Ugurbil, K., Miller, K.L., Smith, S.M., 2014. ICA-based artefact removal and accelerated fMRI acquisition for improved resting state network imaging. NeuroImage 95, 232-247. https://doi.org/10.1016/j.neuroimage.2014.03.034

Gröne, M., Dyck, M., Koush, Y., Bergert, S., Mathiak, K.A., Alawi, E.M., Elliott, M., Mathiak, K., 2015. Upregulation of the Rostral Anterior Cingulate Cortex can Alter the Perception of Emotions: fMRI-Based Neurofeedback at 3 and 7 T. Brain Topogr 28, 197-207. https://doi.org/10.1007/s10548-014-0384-4

Hajnal, J.V., Myers, R., Oatridge, A., Schwieso, J.E., Young, I.R., Bydder, G.M., 1994. Artifacts due to stimulus correlated motion in functional imaging of the brain. Magnetic Resonance in Medicine 31, 283-291. https://doi.org/10.1002/mrm.1910310307

Hamilton, J.P., Glover, G.H., Bagarinao, E., Chang, C., Mackey, S., Sacchet, M.D., Gotlib, I.H., 2016. Effects of saliencenetwork-node neurofeedback training on affective biases in major depressive disorder. Psychiatry Research: Neuroimaging 249, 91-96. https://doi.org/10.1016/j.pscychresns.2016.01.016

Hamilton, J.P., Glover, G.H., Hsu, J.-J., Johnson, R.F., Gotlib, I.H., 2010. Modulation of subgenual anterior cingulate cortex activity with real-time neurofeedback. Human Brain Mapping 32, 22-31. https://doi.org/10.1002/hbm.20997

Handwerker, D.A., Gonzalez-Castillo, J., D’Esposito, M., Bandettini, P.A., 2012. The continuing challenge of understanding and modeling hemodynamic variation in fMRI. Neuroimage 62, 1017-1023. https://doi.org/10.1016/j.neuroimage.2012.02.015

Harmelech, T., Friedman, D., Malach, R., 2015. Differential Magnetic Resonance Neurofeedback Modulations across Extrinsic (Visual) and Intrinsic (Default-Mode) Nodes of the Human Cortex. J. Neurosci. 35, 2588-2595. https://doi.org/10.1523/JNEUROSCI.3098-14.2015

Harmelech, T., Preminger, S., Wertman, E., Malach, R., 2013. The day-after effect: long term, Hebbian-like restructuring of resting-state fMRI patterns induced by a single epoch of cortical activation. J. Neurosci. 33, 9488-9497. https://doi.org/10.1523/JNEUROSCI.5911-12.2013

Hellrung, L., Hollmann, M., Zscheyge, O., Schlumm, T., Kalberlah, C., Roggenhofer, E., Okon-Singer, H., Villringer, A., Horstmann, A., 2015. Flexible Adaptive Paradigms for fMRI Using a Novel Software Package 'Brain Analysis in Real-Time' (BART). PLOS ONE 10, e0118890. https://doi.org/10.1371/journal.pone.0118890

Hellrung, L., Van der Meer, J., Bergert, S., Sladky, R., Pamplona, G.S.P., Scharnowski, F., Koush, Y., Mehler, D.M.A., Linden, D.E.J., Falcon, C., Gispert, J.D., Molinuevo, J.L., Skouras, S., 2017. rtQC: An open-source collaborative framework for quality control methods in real-time fMRI, in: Proceedings of the Real-Time Functional Imaging and Neurofeedback Conference. Nara (Japan).

Heunis, S., Hellrung, L., Van der Meer, J., Bergert, S., Sladky, R., Pamplona, G., Scharnowski, F., Koush, Y., Mehler, D., Falcon, C., Gispert, J., Molinuevo, J., Skouras, S., 2019a. rtQC: An open-source toolbox for real-time fMRI quality control.

Heunis, J.S., Lamerichs, R., Song, G., Zinger, S., Aldenkamp, B., 2019b. Improving BOLD sensitivity with real-time multiecho echo-planar imaging - Towards a cleaner neurofeedback signal. https://doi.org/10.5281/zenodo.2553256 
Hinds, O., Ghosh, S., Thompson, T.W., Yoo, J.J., Whitfield-Gabrieli, S., Triantafyllou, C., Gabrieli, J.D.E., 2011. Computing moment-to-moment BOLD activation for real-time neurofeedback. NeuroImage 54, 361-368. https://doi.org/10.1016/j.neuroimage.2010.07.060

Hirsch, J., Ruge, M.I., Kim, K.H.S., Correa, D.D., Victor, J.D., Relkin, N.R., Labar, D.R., Krol, G., Bilsky, M.H., Souweidane, M.M., DeAngelis, L.M., Gutin, P.H., 2000. An Integrated Functional Magnetic Resonance Imaging Procedure for Preoperative Mapping of Cortical Areas Associated with Tactile, Motor, Language, and Visual Functions: Neurosurgery 47, 711-722. https://doi.org/10.1227/00006123-200009000-00037

Hollmann, M., Mönch, T., Mulla-Osman, S., Tempelmann, C., Stadler, J., Bernarding, J., 2008. A new concept of a unified parameter management, experiment control, and data analysis in fMRI: Application to real-time fMRI at 3T and 7T. Journal of Neuroscience Methods 175, 154-162. https://doi.org/10.1016/j.jneumeth.2008.08.013

Huettel, S.A., McCarthy, G., 2001. Regional Differences in the Refractory Period of the Hemodynamic Response: An EventRelated fMRI Study. NeuroImage 14, 967-976. https://doi.org/10.1006/nimg.2001.0900

Jenkinson, M., Bannister, P., Brady, M., Smith, S., 2002. Improved Optimization for the Robust and Accurate Linear Registration and Motion Correction of Brain Images. NeuroImage 17, 825-841. https://doi.org/10.1006/nimg.2002.1132

Juchem, C., 2013. Shimming: Fields, Coils \& Control, in: Proc. Intl. Soc. Mag. Reson. Med.

Kadosh, K.C., Staunton, G., 2019. A systematic review of the psychological factors that influence neurofeedback learning outcomes. NeuroImage 185, 545-555. https://doi.org/10.1016/j.neuroimage.2018.10.021

Kiebel, S.J., Klöppel, S., Weiskopf, N., Friston, K.J., 2007. Dynamic causal modeling: A generative model of slice timing in fMRI. NeuroImage 34, 1487-1496. https://doi.org/10.1016/j.neuroimage.2006.10.026

Kim, D.-Y., Yoo, S.-S., Tegethoff, M., Meinlschmidt, G., Lee, J.-H., 2015. The inclusion of functional connectivity information into fMRI-based neurofeedback improves its efficacy in the reduction of cigarette cravings. J Cogn Neurosci 27, 1552-1572. https://doi.org/10.1162/jocn a 00802

Kopel, R., Sladky, R., Laub, P., Koush, Y., Robineau, F., Hutton, C., Weiskopf, N., Vuilleumier, P., Van De Ville, D., Scharnowski, F., 2019. No time for drifting: Comparing performance and applicability of signal detrending algorithms for real-time fMRI. NeuroImage 191, 421-429. https://doi.org/10.1016/j.neuroimage.2019.02.058

Koush, Y., Ashburner, J., Prilepin, E., Sladky, R., Zeidman, P., Bibikov, S., Scharnowski, F., Nikonorov, A., De Ville, D.V., 2017a. OpenNFT: An open-source Python/Matlab framework for real-time fMRI neurofeedback training based on activity, connectivity and multivariate pattern analysis. NeuroImage 156, 489-503. https://doi.org/10.1016/j.neuroimage.2017.06.039

Koush, Y., Meskaldji, D.-E., Pichon, S., Rey, G., Rieger, S.W., Linden, D.E.J., Van De Ville, D., Vuilleumier, P., Scharnowski, F., 2017b. Learning Control Over Emotion Networks Through Connectivity-Based Neurofeedback. Cereb Cortex 27, 1193-1202. https://doi.org/10.1093/cercor/bhv311

Koush, Y., Rosa, M.J., Robineau, F., Heinen, K., W. Rieger, S., Weiskopf, N., Vuilleumier, P., Van De Ville, D., Scharnowski, F., 2013. Connectivity-based neurofeedback: Dynamic causal modeling for real-time fMRI. NeuroImage 81, 422-430. https://doi.org/10.1016/j.neuroimage.2013.05.010

Koush, Y., Zvyagintsev, M., Dyck, M., Mathiak, K.A., Mathiak, K., 2012. Signal quality and Bayesian signal processing in neurofeedback based on real-time fMRI. NeuroImage, Neuroergonomics: The human brain in action and at work 59, 478-489. https://doi.org/10.1016/j.neuroimage.2011.07.076

Krüger, G., Glover, G.H., 2001. Physiological noise in oxygenation-sensitive magnetic resonance imaging. Magnetic Resonance in Medicine 46, 631-637. https://doi.org/10.1002/mrm.1240

Kundu, P., Brenowitz, N.D., Voon, V., Worbe, Y., Vértes, P.E., Inati, S.J., Saad, Z.S., Bandettini, P.A., Bullmore, E.T., 2013. Integrated strategy for improving functional connectivity mapping using multiecho fMRI. PNAS 110, 1618716192. https://doi.org/10.1073/pnas.1301725110

Kundu, P., Inati, S.J., Evans, J.W., Luh, W.-M., Bandettini, P.A., 2012. Differentiating BOLD and non-BOLD signals in fMRI time series using multi-echo EPI. NeuroImage 60, 1759-1770. https://doi.org/10.1016/j.neuroimage.2011.12.028

Kundu, P., Voon, V., Balchandani, P., Lombardo, M.V., Poser, B.A., Bandettini, P.A., 2017. Multi-echo fMRI: A review of applications in fMRI denoising and analysis of BOLD signals. NeuroImage, Cleaning up the fMRI time series: Mitigating noise with advanced acquisition and correction strategies 154, 59-80. https://doi.org/10.1016/j.neuroimage.2017.03.033

LaConte, S.M., 2011. Decoding fMRI brain states in real-time. NeuroImage 56, 440-454. https://doi.org/10.1016/j.neuroimage.2010.06.052

LaConte, S.M., Peltier, S.J., Hu, X.P., 2007a. Real-time fMRI using brain-state classification. Human Brain Mapping 28, 1033-1044. https://doi.org/10.1002/hbm.20326

LaConte, S.M., Peltier, S.J., Hu, X.P., 2007b. Real-time fMRI using brain-state classification. Human Brain Mapping 28, 1033-1044. https://doi.org/10.1002/hbm.20326 
Linden, D.E.J., Habes, I., Johnston, S.J., Linden, S., Tatineni, R., Subramanian, L., Sorger, B., Healy, D., Goebel, R., 2012. Real-Time Self-Regulation of Emotion Networks in Patients with Depression. PLOS ONE 7, e38115. https://doi.org/10.1371/journal.pone.0038115

Liu, T.T., 2016. Noise contributions to the fMRI signal: An overview. NeuroImage 143, 141-151. https://doi.org/10.1016/j.neuroimage.2016.09.008

Logothetis, N.K., 2003. The Underpinnings of the BOLD Functional Magnetic Resonance Imaging Signal. J. Neurosci. 23, 3963-3971. https://doi.org/10.1523/JNEUROSCI.23-10-03963.2003

Maclaren, J., Herbst, M., Speck, O., Zaitsev, M., 2012. Prospective motion correction in brain imaging: A review. Magnetic Resonance in Medicine 69, 621-636. https://doi.org/10.1002/mrm.24314

Marins, T.F., Rodrigues, E.C., Engel, A., Hoefle, S., Basílio, R., Lent, R., Moll, J., Tovar-Moll, F., 2015. Enhancing Motor Network Activity Using Real-Time Functional MRI Neurofeedback of Left Premotor Cortex. Front. Behav. Neurosci. 9. https://doi.org/10.3389/fnbeh.2015.00341

Marxen, M., Jacob, M.J., Müller, D.K., Posse, S., Ackley, E., Hellrung, L., Riedel, P., Bender, S., Epple, R., Smolka, M.N., 2016. Amygdala Regulation Following fMRI-Neurofeedback without Instructed Strategies. Front. Hum. Neurosci. 10 https://doi.org/10.3389/fnhum.2016.00183

Mathiak, K., Posse, S., 2001. Evaluation of motion and realignment for functional magnetic resonance imaging in real time. Magnetic Resonance in Medicine 45, 167-171. https://doi.org/10.1002/1522-2594(200101)45:1<167::AIDMRM1023>3.0.CO;2-M

Mathiak, K., Rapp, A., Kircher, T.T.J., Grodd, W., Hertrich, I., Weiskopf, N., Lutzenberger, W., Ackermann, H., 2002. Mismatch responses to randomized gradient switching noise as reflected by fMRI and whole-head magnetoencephalography. Human Brain Mapping 16, 190-195. https://doi.org/10.1002/hbm.10041

Mazaika, P.K., Hoeft, F., Glover, G.H., Reiss, A.L., 2009. Methods and software for fMRI analysis for clinical subjects. Presented at the Presentation at the 15th Annual Meeting of the Organization for Human Brain Mapping.

McCaig, R.G., Dixon, M., Keramatian, K., Liu, I., Christoff, K., 2011. Improved modulation of rostrolateral prefrontal cortex using real-time fMRI training and meta-cognitive awareness. NeuroImage 55, 1298-1305. https://doi.org/10.1016/j.neuroimage.2010.12.016

McDonald, A.R., Muraskin, J., Van Dam, N.T., Froehlich, C., Puccio, B., Pellman, J., Bauer, C.C., Akeyson, A., Breland, M.M., Calhoun, V.D., Carter, S., Chang, T.P., Gessner, C., Gianonne, A., Giavasis, S., Glass, J., Homan, S., King, M., Kramer, M., Landis, D., Lieval, A., Lisinski, J., Mackay-Brandt, A., Miller, B., Panek, L., Reed, H., Santiago, C., Schoell, E., Sinnig, R., Sital, M., Taverna, E., Tobe, R., Trautman, K., Varghese, B., Walden, L., Wang, R., Waters, A.B., Wood, D., Castellanos, F.X., Leventhal, B., Colcombe, S.J., LaConte, S., Milham, M.P., Craddock, R.C., 2017. The Real-time fMRI Neurofeedback Based Stratification of Default Network Regulation Neuroimaging Data Repository. Neuroimage 146, 157-170. https://doi.org/10.1016/j.neuroimage.2016.10.048

Misaki, M., Barzigar, N., Zotev, V., Phillips, R., Cheng, S., Bodurka, J., 2015. Real-time fMRI processing with physiological noise correction - Comparison with off-line analysis. Journal of Neuroscience Methods 256, 117-121. https://doi.org/10.1016/j.jneumeth.2015.08.033

Morawetz, C., Holz, P., Lange, C., Baudewig, J., Weniger, G., Irle, E., Dechent, P., 2008. Improved functional mapping of the human amygdala using a standard functional magnetic resonance imaging sequence with simple modifications. Magnetic Resonance Imaging 26, 45-53. https://doi.org/10.1016/j.mri.2007.04.014

Munafò, M.R., Nosek, B.A., Bishop, D.V.M., Button, K.S., Chambers, C.D., Sert, N.P. du, Simonsohn, U., Wagenmakers, E.-J., Ware, J.J., Ioannidis, J.P.A., 2017. A manifesto for reproducible science. Nature Human Behaviour 1, 0021. https://doi.org/10.1038/s41562-016-0021

Murphy, K., Birn, R.M., Bandettini, P.A., 2013. Resting-state FMRI confounds and cleanup. Neuroimage 80, 349-359. https://doi.org/10.1016/j.neuroimage.2013.04.001

Murphy, K., Birn, R.M., Handwerker, D.A., Jones, T.B., Bandettini, P.A., 2009. The impact of global signal regression on resting state correlations: Are anti-correlated networks introduced? NeuroImage 44, 893-905. https://doi.org/10.1016/j.neuroimage.2008.09.036

Murphy, K., Bodurka, J., Bandettini, P.A., 2007. How long to scan? The relationship between fMRI temporal signal to noise and necessary scan duration. Neuroimage 34, 565-574. https://doi.org/10.1016/j.neuroimage.2006.09.032

Murphy, K., Fox, M.D., 2017. Towards a consensus regarding global signal regression for resting state functional connectivity MRI. Neuroimage 154, 169-173. https://doi.org/10.1016/j.neuroimage.2016.11.052

Nichols, T.E., Das, S., Eickhoff, S.B., Evans, A.C., Glatard, T., Hanke, M., Kriegeskorte, N., Milham, M.P., Poldrack, R.A., Poline, J.-B., Proal, E., Thirion, B., Essen, D.C.V., White, T., Yeo, B.T.T., 2017. Best practices in data analysis and sharing in neuroimaging using MRI [WWW Document]. Nature Neuroscience. https://doi.org/10.1038/nn.4500

Noll, D.C., Schneider, W., 1994. Theory, simulation, and compensation of physiological motion artifacts in functional MRI, in: Proceedings of 1st International Conference on Image Processing. Presented at the Proceedings of 1st International Conference on Image Processing, pp. 40-44 vol.3. https://doi.org/10.1109/ICIP.1994.413892 
Ogawa, S., Menon, R.S., Kim, S.-G., Ugurbil, and K., 1998. On the Characteristics of Functional Magnetic Resonance Imaging of the Brain. Annual Review of Biophysics and Biomolecular Structure 27, 447-474. https://doi.org/10.1146/annurev.biophys.27.1.447

Parkes, L., Fulcher, B., Yücel, M., Fornito, A., 2018. An evaluation of the efficacy, reliability, and sensitivity of motion correction strategies for resting-state functional MRI. NeuroImage 171, 415-436. https://doi.org/10.1016/j.neuroimage.2017.12.073

Parrish, T.B., Gitelman, D.R., LaBar, K.S., Mesulam, M.-M., 2000. Impact of signal-to-noise on functional MRI. Magnetic Resonance in Medicine 44, 925-932. https://doi.org/10.1002/1522-2594(200012)44:6<925::AID-MRM14>3.0.CO;2$\mathrm{M}$

Perlbarg, V., Bellec, P., Anton, J.-L., Pélégrini-Issac, M., Doyon, J., Benali, H., 2007. CORSICA: correction of structured noise in fMRI by automatic identification of ICA components. Magnetic Resonance Imaging 25, 35-46. https://doi.org/10.1016/j.mri.2006.09.042

Poldrack, R.A., Mumford, J.A., Nichols, T.E., 2011. Handbook of Functional MRI Data Analysis [WWW Document]. Cambridge Core. https://doi.org/10.1017/CBO9780511895029

Poser, B.A., Versluis, M.J., Hoogduin, J.M., Norris, D.G., 2006. BOLD contrast sensitivity enhancement and artifact reduction with multiecho EPI: Parallel-acquired inhomogeneity-desensitized fMRI. Magnetic Resonance in Medicine 55, 1227-1235. https://doi.org/10.1002/mrm.20900

Posse, S., Ackley, E., Mutihac, R., Rick, J., Shane, M., Murray-Krezan, C., Zaitsev, M., Speck, O., 2012. Enhancement of temporal resolution and BOLD sensitivity in real-time fMRI using multi-slab echo-volumar imaging. NeuroImage 61, 115-130. https://doi.org/10.1016/j.neuroimage.2012.02.059

Posse, S., Binkofski, F., Schneider, F., Gembris, D., Frings, W., Habel, U., Salloum, J.B., Mathiak, K., Wiese, S., Kiselev, V., Graf, T., Elghahwagi, B., Grosse-Ruyken, M.-L., Eickermann, T., 2000. A new approach to measure single-event related brain activity using real-time fMRI: Feasibility of sensory, motor, and higher cognitive tasks. Human Brain Mapping 12, 25-41. https://doi.org/10.1002/1097-0193(200101)12:1<25::AID-HBM30>3.0.CO;2-H

Posse, S., Shen, Z., Kiselev, V., Kemna, L.J., 2003. Single-shot T2* mapping with 3D compensation of local susceptibility gradients in multiple regions. NeuroImage 18, 390-400. https://doi.org/10.1016/S1053-8119(02)00016-2

Posse, S., Wiese, S., Gembris, D., Mathiak, K., Kessler, C., Grosse-Ruyken, M.-L., Elghahwagi, B., Richards, T., Dager, S.R., Kiselev, V.G., 1999. Enhancement of BOLD-contrast sensitivity by single-shot multi-echo functional MR imaging. Magnetic Resonance in Medicine 42, 87-97. https://doi.org/10.1002/(SICI)15222594(199907)42:1<87::AID-MRM13>3.0.CO;2-O

Posse, S., Wiese, S., Kessler, C., Gembris, D., Weiss, U., Peyerl, M., Grosse-Ruyken, M., Elghawaghi, B., Richards, T., Dager, S., 1998. Single Shot T2*-Sensitive Spectroscopic Imaging Increases fMRI Sensitivity: Preliminary Evidence from Visual and Olfactory Activation., in: International Society for Magnetic Resonance in Medicine.

Power, J.D., 2017. A simple but useful way to assess fMRI scan qualities. NeuroImage, Cleaning up the fMRI time series: Mitigating noise with advanced acquisition and correction strategies 154, 150-158. https://doi.org/10.1016/j.neuroimage.2016.08.009

Power, J.D., Barnes, K.A., Snyder, A.Z., Schlaggar, B.L., Petersen, S.E., 2012. Spurious but systematic correlations in functional connectivity MRI networks arise from subject motion. Neuroimage 59, 2142-2154. https://doi.org/10.1016/j.neuroimage.2011.10.018

Power, J.D., Mitra, A., Laumann, T.O., Snyder, A.Z., Schlaggar, B.L., Petersen, S.E., 2014. Methods to detect, characterize, and remove motion artifact in resting state fMRI. Neuroimage 84. https://doi.org/10.1016/j.neuroimage.2013.08.048

Power, J.D., Plitt, M., Gotts, S.J., Kundu, P., Voon, V., Bandettini, P.A., Martin, A., 2018. Ridding fMRI data of motionrelated influences: Removal of signals with distinct spatial and physical bases in multiecho data. PNAS 201720985. https://doi.org/10.1073/pnas.1720985115

Power, J.D., Plitt, M., Laumann, T.O., Martin, A., 2017. Sources and implications of whole-brain fMRI signals in humans. Neuroimage 146, 609-625. https://doi.org/10.1016/j.neuroimage.2016.09.038

Processed Connectomes Project, 2014. PCP Quality Assessment Protocol. [ONLINE] Available at: http://preprocessedconnectomes-project.org/quality-assessment-protocol/. [Accessed 28 May 2018].

Ramot, M., Gonzalez-Castillo, J., 2019. A framework for offline evaluation and optimization of real-time algorithms for use in neurofeedback, demonstrated on an instantaneous proxy for correlations. NeuroImage 188, 322-334. https://doi.org/10.1016/j.neuroimage.2018.12.006

Rangaprakash, D., Wu, G.-R., Marinazzo, D., Hu, X., Deshpande, G., 2018. Hemodynamic response function (HRF) variability confounds resting-state fMRI functional connectivity. Magnetic Resonance in Medicine 0. https://doi.org/10.1002/mrm.27146

Ruiz, S., Lee, S., Soekadar, S.R., Caria, A., Veit, R., Kircher, T., Birbaumer, N., Sitaram, R., 2013. Acquired self-control of insula cortex modulates emotion recognition and brain network connectivity in schizophrenia. Human Brain Mapping 34, 200-212. https://doi.org/10.1002/hbm.21427 
Scheinost, D., Hampson, M., Qiu, M., Bhawnani, J., Constable, R.T., Papademetris, X., 2013. A Graphics Processing Unit Accelerated Motion Correction Algorithm and Modular System for Real-time fMRI. Neuroinform 11, 291-300. https://doi.org/10.1007/s12021-013-9176-3

Shibata, K., Watanabe, T., Kawato, M., Sasaki, Y., 2016. Differential Activation Patterns in the Same Brain Region Led to Opposite Emotional States. PLOS Biology 14, e1002546. https://doi.org/10.1371/journal.pbio.1002546

Shibata, K., Watanabe, T., Sasaki, Y., Kawato, M., 2011. Perceptual Learning Incepted by Decoded fMRI Neurofeedback Without Stimulus Presentation. Science 334, 1413-1415. https://doi.org/10.1126/science.1212003

Siegel, J.S., Power, J.D., Dubis, J.W., Vogel, A.C., Church, J.A., Schlaggar, B.L., Petersen, S.E., 2014. Statistical improvements in functional magnetic resonance imaging analyses produced by censoring high-motion data points. Human Brain Mapping 35, 1981-1996. https://doi.org/10.1002/hbm.22307

Sitaram, R., Ros, T., Stoeckel, L., Haller, S., Scharnowski, F., Lewis-Peacock, J., Weiskopf, N., Blefari, M.L., Rana, M., Oblak, E., Birbaumer, N., Sulzer, J., 2017. Closed-loop brain training: the science of neurofeedback. Nature Reviews Neuroscience 18, 86-100. https://doi.org/10.1038/nrn.2016.164

Skinner, B.F., 1945. The operational analysis of psychological terms. Psychological Review 52, $268-277$.

Sladky, R., Friston, K.J., Tröstl, J., Cunnington, R., Moser, E., Windischberger, C., 2011. Slice-timing effects and their correction in functional MRI. NeuroImage 58, 588-594. https://doi.org/10.1016/j.neuroimage.2011.06.078

Smith, A.M., Lewis, B.K., Ruttimann, U.E., Ye, F.Q., Sinnwell, T.M., Yang, Y., Duyn, J.H., Frank, J.A., 1999. Investigation of Low Frequency Drift in fMRI Signal. NeuroImage 9, 526-533. https://doi.org/10.1006/nimg.1999.0435

Smyser, C., Grabowski, T.J., Frank, R.J., Haller, J.W., Bolinger, L., 2001. Real-time multiple linear regression for fMRI supported by time-aware acquisition and processing. Magnetic Resonance in Medicine 45, 289-298. https://doi.org/10.1002/1522-2594(200102)45:2<289::AID-MRM1038>3.0.CO;2-U

Sorger, B., Kamp, T., Weiskopf, N., Peters, J.C., Goebel, R., 2018. When the Brain Takes 'BOLD' Steps: Real-Time fMRI Neurofeedback Can Further Enhance the Ability to Gradually Self-regulate Regional Brain Activation. Neuroscience, Neurofeedback and Functional Enhancement: Mechanisms, Methodology, Behavioral and Clinical Applications 378, 71-88. https://doi.org/10.1016/j.neuroscience.2016.09.026

Sorger, B., Reithler, J., Dahmen, B., Goebel, R., 2012. A Real-Time fMRI-Based Spelling Device Immediately Enabling Robust Motor-Independent Communication. Current Biology 22, 1333-1338. https://doi.org/10.1016/j.cub.2012.05.022

Spetter, M.S., Malekshahi, R., Birbaumer, N., Lührs, M., van der Veer, A.H., Scheffler, K., Spuckti, S., Preissl, H., Veit, R., Hallschmid, M., 2017. Volitional regulation of brain responses to food stimuli in overweight and obese subjects: A real-time fMRI feedback study. Appetite 112, 188-195. https://doi.org/10.1016/j.appet.2017.01.032

Stoeckel, L.E., Garrison, K.A., Ghosh, S.S., Wighton, P., Hanlon, C.A., Gilman, J.M., Greer, S., Turk-Browne, N.B., deBettencourt, M.T., Scheinost, D., Craddock, C., Thompson, T., Calderon, V., Bauer, C.C., George, M., Breiter, H.C., Whitfield-Gabrieli, S., Gabrieli, J.D., LaConte, S.M., Hirshberg, L., Brewer, J.A., Hampson, M., Van Der Kouwe, A., Mackey, S., Evins, A.E., 2014. Optimizing real time fMRI neurofeedback for therapeutic discovery and development. NeuroImage: Clinical 5, 245-255. https://doi.org/10.1016/j.nicl.2014.07.002

Sulzer, J., Haller, S., Scharnowski, F., Weiskopf, N., Birbaumer, N., Blefari, M.L., Bruehl, A.B., Cohen, L.G., deCharms, R.C., Gassert, R., Goebel, R., Herwig, U., LaConte, S., Linden, D., Luft, A., Seifritz, E., Sitaram, R., 2013. Real-time fMRI neurofeedback: Progress and challenges. NeuroImage 76, 386-399. https://doi.org/10.1016/j.neuroimage.2013.03.033

Sulzer, James, Sitaram, R., Blefari, M.L., Kollias, S., Birbaumer, N., Stephan, K.E., Luft, A., Gassert, R., 2013. Neurofeedback-mediated self-regulation of the dopaminergic midbrain. NeuroImage 83, 817-825. https://doi.org/10.1016/j.neuroimage.2013.05.115

Thibault, R.T., MacPherson, A., Lifshitz, M., Roth, R.R., Raz, A., 2018. Neurofeedback with fMRI: A critical systematic review. NeuroImage 172, 786-807. https://doi.org/10.1016/j.neuroimage.2017.12.071

Triantafyllou, C., Hoge, R.D., Krueger, G., Wiggins, C.J., Potthast, A., Wiggins, G.C., Wald, L.L., 2005. Comparison of physiological noise at $1.5 \mathrm{~T}, 3 \mathrm{~T}$ and $7 \mathrm{~T}$ and optimization of fMRI acquisition parameters. NeuroImage 26, $243-250$. https://doi.org/10.1016/j.neuroimage.2005.01.007

Van Dijk, K.R.A., Sabuncu, M.R., Buckner, R.L., 2012. The influence of head motion on intrinsic functional connectivity MRI. NeuroImage, Neuroergonomics: The human brain in action and at work 59, 431-438. https://doi.org/10.1016/j.neuroimage.2011.07.044

Vidaurre, C., Blankertz, B., 2010. Towards a Cure for BCI Illiteracy. Brain Topogr 23, 194-198. https://doi.org/10.1007/s10548-009-0121-6

Voyvodic, 2011. Automated real-time behavioral and physiological data acquisition and display integrated with stimulus presentation for fMRI. Frontiers in Neuroinformatics. https://doi.org/10.3389/fninf.2011.00027

Voyvodic, J.T., 1999. Real-Time fMRI Paradigm Control, Physiology, and Behavior Combined with Near Real-Time Statistical Analysis. NeuroImage 10, 91-106. https://doi.org/10.1006/nimg.1999.0457 
Ward, H.A., Riederer, S.J., Jack, C.R., 2002. Real-time autoshimming for echo planar timecourse imaging. Magnetic Resonance in Medicine 48, 771-780. https://doi.org/10.1002/mrm.10259

Watanabe, T., Sasaki, Y., Shibata, K., Kawato, M., 2017. Advances in fMRI Real-Time Neurofeedback. Trends in Cognitive Sciences 21, 997-1010. https://doi.org/10.1016/j.tics.2017.09.010

Weiskopf, N., Hutton, C., Josephs, O., Deichmann, R., 2006. Optimal EPI parameters for reduction of susceptibility-induced BOLD sensitivity losses: A whole-brain analysis at $3 \mathrm{~T}$ and $1.5 \mathrm{~T}$. NeuroImage 33, 493-504. https://doi.org/10.1016/j.neuroimage.2006.07.029

Weiskopf, N., Hutton, C., Josephs, O., Turner, R., Deichmann, R., 2007a. Optimized EPI for fMRI studies of the orbitofrontal cortex: compensation of susceptibility-induced gradients in the readout direction. Magnetic Resonance Materials in Physics, Biology and Medicine 20, 39-49. https://doi.org/10.1007/s10334-006-0067-6

Weiskopf, N., Klose, U., Birbaumer, N., Mathiak, K., 2005. Single-shot compensation of image distortions and BOLD contrast optimization using multi-echo EPI for real-time fMRI. NeuroImage 24, 1068-1079. https://doi.org/10.1016/j.neuroimage.2004.10.012

Weiskopf, N., Mathiak, K., Bock, S.W., Scharnowski, F., Veit, R., Grodd, W., Goebel, R., Birbaumer, N., 2004. Principles of a brain-computer interface (BCI) based on real-time functional magnetic resonance imaging (fMRI). IEEE Transactions on Biomedical Engineering 51, 966-970. https://doi.org/10.1109/TBME.2004.827063

Weiskopf, Nikolaus, Scharnowski, F., Veit, R., Goebel, R., Birbaumer, N., Mathiak, K., 2004. Self-regulation of local brain activity using real-time functional magnetic resonance imaging (fMRI). Journal of Physiology-Paris, Decoding and interfacing the brain: from neuronal assemblies to cyborgs 98, 357-373. https://doi.org/10.1016/j.jphysparis.2005.09.019

Weiskopf, N., Sitaram, R., Josephs, O., Veit, R., Scharnowski, F., Goebel, R., Birbaumer, N., Deichmann, R., Mathiak, K., 2007b. Real-time functional magnetic resonance imaging: methods and applications. Magnetic Resonance Imaging 25, 989-1003. https://doi.org/10.1016/j.mri.2007.02.007

Welvaert, M., Rosseel, Y., 2013. On the Definition of Signal-To-Noise Ratio and Contrast-To-Noise Ratio for fMRI Data. PLOS ONE 8, e77089. https://doi.org/10.1371/journal.pone.0077089

Whitfield-Gabrieli, S., Nieto-Castanon, A., 2012. Conn: A Functional Connectivity Toolbox for Correlated and Anticorrelated Brain Networks. Brain Connectivity 2, 125-141. https://doi.org/10.1089/brain.2012.0073

Wilms, M., Schilbach, L., Pfeiffer, U., Bente, G., Fink, G.R., Vogeley, K., 2010. It's in your eyes-using gaze-contingent stimuli to create truly interactive paradigms for social cognitive and affective neuroscience. Soc Cogn Affect Neurosci 5, 98-107. https://doi.org/10.1093/scan/nsq024

Wise, R.G., Ide, K., Poulin, M.J., Tracey, I., 2004. Resting fluctuations in arterial carbon dioxide induce significant low frequency variations in BOLD signal. NeuroImage 21, 1652-1664. https://doi.org/10.1016/j.neuroimage.2003.11.025

Yamashita, A., Hayasaka, S., Kawato, M., Imamizu, H., 2017. Connectivity Neurofeedback Training Can Differentially Change Functional Connectivity and Cognitive Performance. Cereb. Cortex 27, 4960-4970. https://doi.org/10.1093/cercor/bhx177

Yang, S., Ross, T.J., Zhang, Y., Stein, E.A., Yang, Y., 2005. Head motion suppression using real-time feedback of motion information and its effects on task performance in fMRI. NeuroImage 27, 153-162. https://doi.org/10.1016/j.neuroimage.2005.02.050

Yoo, S.-S., Jolesz, F.A., 2002. Functional MRI for neurofeedback: feasibility study on a hand motor task. Neuroreport 13, 1377-1381.

Young, K.D., Zotev, V., Phillips, R., Misaki, M., Yuan, H., Drevets, W.C., Bodurka, J., 2014. Real-Time fMRI Neurofeedback Training of Amygdala Activity in Patients with Major Depressive Disorder. PLOS ONE 9, e88785. https://doi.org/10.1371/journal.pone.0088785

Zaitsev, M., Hennig, J., Speck, O., 2004. Point spread function mapping with parallel imaging techniques and high acceleration factors: Fast, robust, and flexible method for echo-planar imaging distortion correction. Magnetic Resonance in Medicine 52, 1156-1166. https://doi.org/10.1002/mrm.20261

Zhuo, J., Gullapalli, R.P., 2006. MR Artifacts, Safety, and Quality Control. RadioGraphics 26, 275-297. https://doi.org/10.1148/rg.261055134

Zilverstand, A., Sorger, B., Slaats-Willemse, D., Kan, C.C., Goebel, R., Buitelaar, J.K., 2017. fMRI Neurofeedback Training for Increasing Anterior Cingulate Cortex Activation in Adult Attention Deficit Hyperactivity Disorder. An Exploratory Randomized, Single-Blinded Study. PLOS ONE 12, e0170795. https://doi.org/10.1371/journal.pone.0170795

Zilverstand, A., Sorger, B., Zimmermann, J., Kaas, A., Goebel, R., 2014a. Windowed Correlation: A Suitable Tool for Providing Dynamic fMRI-Based Functional Connectivity Neurofeedback on Task Difficulty. PLOS ONE 9, e85929. https://doi.org/10.1371/journal.pone.0085929

Zilverstand, A., Sorger, B., Zimmermann, J., Kaas, A., Goebel, R., 2014b. Windowed Correlation: A Suitable Tool for Providing Dynamic fMRI-Based Functional Connectivity Neurofeedback on Task Difficulty. PLOS ONE 9, e85929. https://doi.org/10.1371/journal.pone.0085929 
